\title{
Prospects and Applications Near Ferroelectric Quantum Phase Transitions: A Key Issues Review
}

\author{
P. Chandra, ${ }^{1}$ G.G. Lonzarich, ${ }^{2}$ S.E. Rowley, ${ }^{2,3}$ and J.F. Scott ${ }^{2,4} \ddagger$ \\ ${ }^{1}$ Center for Materials Theory, Department of Physics and Astronomy, Rutgers \\ University, Piscataway, NJ 08854 USA. \\ ${ }^{2}$ Cavendish Laboratory, Cambridge University, J.J. Thomson Avenue, Cambridge \\ CB3 0HE UK. \\ ${ }^{3}$ Centro Brasileiro de Pesquisas Fisicas, Rua Dr. Xavier Sigaud 150, Rio de Janeiro \\ 22290-180, Brazil. \\ ${ }^{4}$ Department of Physics, St. Andrews University, Scotland UK.
}

\begin{abstract}
The emergence of complex and fascinating states of quantum matter in the neighborhood of zero temperature phase transitions suggests that such quantum phenomena should be studied in a variety of settings. Advanced technologies of the future may be fabricated from materials where the cooperative behavior of charge, spin and current can be manipulated at cryogenic temperatures. The progagating lattice dynamics of displacive ferroelectrics make them appealing for the study of quantum critical phenomena that is characterized by both space- and time-dependent quantities. In this Key Issues article we aim to provide a self-contained overview of ferroelectrics near quantum phase transitions. Unlike most magnetic cases, the ferroelectric quantum critical point can be tuned experimentally to reside at, above or below its upper critical dimension; this feature allows for detailed interplay between experiment and theory using both scaling and self-consistent field models. Empirically the sensitivity of the ferroelectric $T_{c}$ 's to external and to chemical pressure gives practical access to a broad range of temperature behavior over several hundreds of Kelvin. Additional degrees of freedom like charge and spin can be added and characterized systematically. Satellite memories, electrocaloric cooling and low-loss phased-array radar are among possible applications of low-temperature ferroelectrics. We end with open questions for future research that include textured polarization states and unusual forms of superconductivity that remain to be understood theoretically.
\end{abstract}

Submitted to: Rep. Prog. Phys. (invited)

$\ddagger$ The alphabetical ordering of the authors indicates they contributed equally to this article 


\section{Contents}

1 Introduction and FAQs 2

2 Quantum Criticality Basics $\quad 10$

3 Ferroelectrics Necessities $\quad 16$

4 The Case of $\mathrm{SrTiO}_{3}$ to Date $\quad 24$

5 A Flavor for Low Temperature Applications $\quad 29$

6 Open Questions for Future Research 35

$\begin{array}{lll}7 & \text { Acknowledgements } & 40\end{array}$

8 References $\quad 41$

\section{Introduction and FAQs}

At first sight, the links between ferroelectrics, quantum phase transitions and quantum criticality may not be obvious. After all, ferroelectrics are mostly non-metallic materials that are often studied towards specific functionalities at room temperature, whereas a key motivation for research in quantum phase transitions and quantum criticality is their links with novel metallic behavior and exotic superconductivity. Our principal aim in this Key Issues article is to encourage more communication between researchers in these two mainly independent communities. Let us begin by addressing frequently asked questions that might be posed by curious newcomers to the field in a colloquial fashion before presenting more detail in the subsequent parts of this article.

$* * * * * * * * * * * * * * * * * * * * * * * * * * * * * * * *$

\section{Aren't quantum fluctuations only important at $T=0$ Kelvin ?}

Let's start by discussing what is meant by quantum fluctuations. We can begin by thinking about the amplitude fluctuations of a one-dimensional simple harmonic oscillator as a function of temperature, and let's take a look at Figure 1 together. Here we see that, setting the constants $\hbar$ and $k_{B}$ to be unity, the important energy-scales are the temperature, $T$, and the oscillator frequency $\Omega$. If $T$ is much greater than $\Omega$, then the variance in the amplitude, $\left\langle x^{2}\right\rangle$, scales with $T$ and $\Omega$ drops out completely. In this case, the total variance results from purely classical (thermal) fluctuations and in Figure 1 their contribution to $\left\langle x^{2}\right\rangle$ is indicated by a red line. However for lower temperatures, particularly in the interval $0<T \lesssim \Omega$, there is another contribution to $\left\langle x^{2}\right\rangle$ above this classical red line (see Figure 1) due to quantum fluctuations (blue line in Figure 1). 
The total variance then becomes the sum of the quantum and the classical components, where at $T=0$ only the quantum component survives.

\section{Simple Harmonic Oscillator}

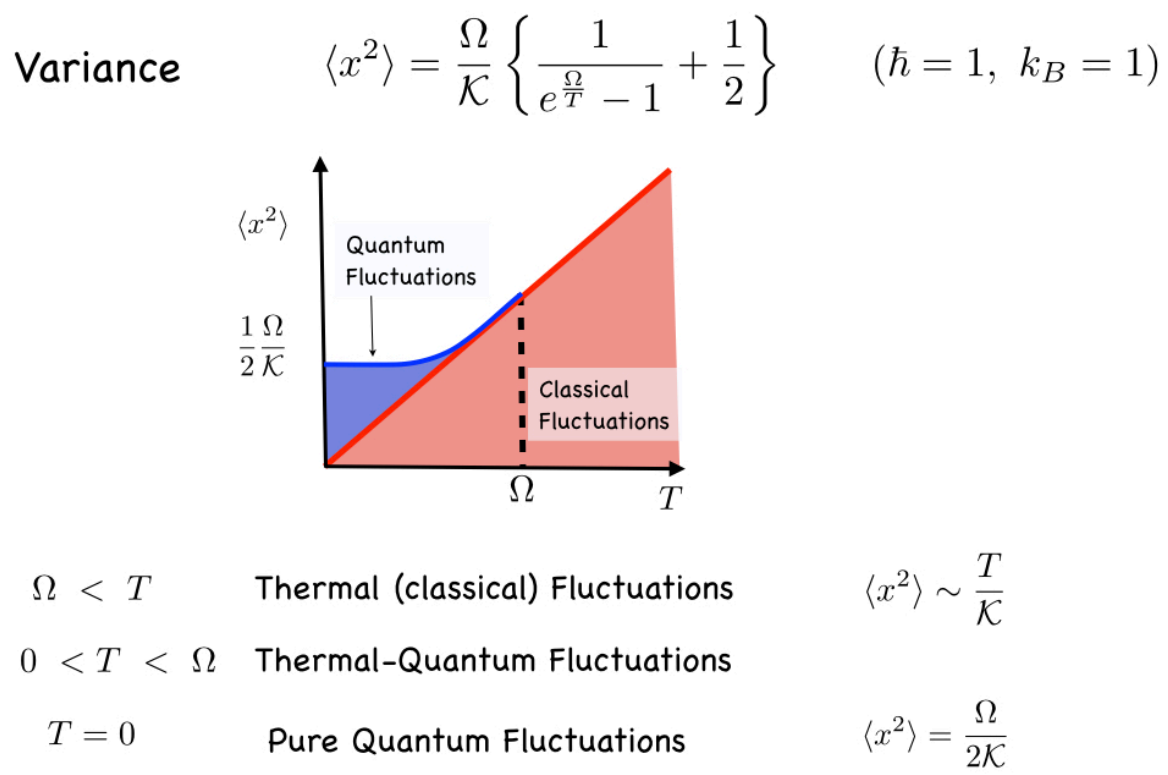

Figure 1. Amplitude variance of a simple harmonic oscillator where $\Omega$ and $\mathcal{K}$ are its frequency and stiffness respectively.

Fine, but what does this behavior of one simple harmonic oscillator have to do with quantum phase transitions?

We are just getting to this conceptual connection. Order parameter fluctuations play a key role at phase transitions, and we can consider the variance of each of their Fourier components one at a time. We can call each of these Fourier components a mode of wavevector $q$ whose behavior could be mapped onto that of single harmonic oscillator of amplitude $x$ where $\Omega$ would be the oscillator frequency of the mode in question. Now we are back to our Figure 1 where the full variance $\left\langle x^{2}\right\rangle$ is plotted as a function of temperature for a particular mode of wavevector $q$. At a continuous phase transition the (mode) stiffness $\mathcal{K}$ vanishes for modes with wavevectors close to the ordering wavevector, so that the red and blue lines in Figure 1 becomes vertical and the amplitude fluctuations diverge. If this occurs at a temperature $T \gg \Omega$, then the transition may be driven by essentially classical fluctuations and is between high to low entropy states as a function of decreasing temperature. However at low temperatures where $0<T \lesssim \Omega$, we have classical-plus-quantum $(\mathrm{C}+\mathrm{Q})$ fluctuations and here we are very interested in how these "hybrid" fluctuations lead to behavior and ordering distinct from those driven by their purely classical counterparts. Again $\mathcal{K}$ at the ordering wavevector goes 
to zero at the transition but now, in addition to the classical contribution, there is a quantum component to $\left\langle x^{2}\right\rangle$. Of course at strictly $T=0$ the fluctuations are purely quantum and the entropy change is zero for an equilibrium system. Therefore purely (equilibrium) quantum phase transitions are really transformations from one type of ordering to another. We emphasize this point because the term "quantum disordered state", that often appears in phase diagrams, is ambiguous and possibly confusing; it may only have useful meaning in cases where there is a finite ground-state degeneracy in violation of the Third Law of thermodynamics.

Here you are telling us that quantum fluctuations increase amplitude fluctuations at low temperatures. However Einstein and later Debye showed that quantum fluctuations reduced the specific heat from its classical value and that was a big success for the quantum theory. How does this fit in with what you are saying?

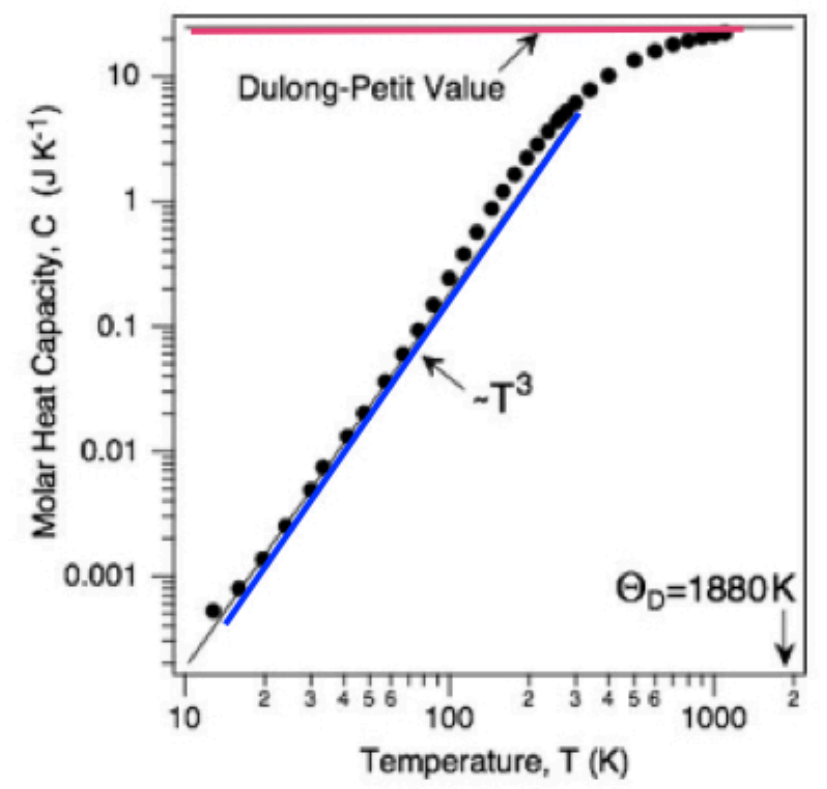

Figure 2. Heat capacity of diamond vs. temperature. Note that at room temperature it is well below the classical Dulong-Petit value, indicating the importance of quantum effects at non-cryogenic temperatures. Adapted from Hofman [1] with permission and thanks to P. Hofman.

You are of course completely correct that at low temperatures the specific heat of a solid is reduced compared to its classical constant value, and indeed this may seem counterintuitive given what we've just told you. However we can in fact understand this behavior by looking again at Figure 1. In our simple approach the energy is proportional to the variance of amplitude fluctuations, so the specific heat is then its derivative. We 
see that the slope of the variance in the amplitude $\left(\left\langle x^{2}\right\rangle\right)$ is higher at temperatures $T>>\Omega$ than at $T<<\Omega$, and indeed it is actually relatively flat in the approach to zero-temperature. This means that the specific heat will be significantly lower at low temperatures compared to its constant value at temperatures $T>>\Omega$ and we hope this answers the question. In Figure 2 you see the specific heat $c_{P}$ of diamond that has a Debye temperature exceeding one thousand degrees (Kelvin); at room temperature $c_{P}$ is already temperature-dependent and thus the effects of quantum fluctuations are observable without any fancy cryogenics!

As you suggest, the heat capacity is valuable in bringing out the dramatic quantum corrections to classical behavior that can extend to room temperatures and above. However it is also important to note that the heat capacity does not reflect the total variance and depends only on the Bose function contribution; of course we are neglecting any temperature-dependence of $\Omega$ which would require a more extended discussion.

\section{So then why do we care about the total variance anyway if it isn't important for observable quantities?}

We agree that this is not obvious from our specific heat discussion. As we can see in Figure 1, the total variance has both classical and quantum components, where their relative contributions change as a function of temperature. Just as the classical part drives phase transitions for $T \gg \Omega$, it is the quantum part that drives phase transitions for $T \ll \Omega$. We should add that the total variance of the amplitude fluctuations can be probed, for example, by neutron scattering experiments where the neutron loses energy to the system so that both the zero-point and the Bose function contributions are measured. Again we stress that it is the total variance that is crucial for the "disruption" of the initial form of order.

\section{What does quantum criticality mean?}

In a nutshell, quantum criticality refers to a second-order phase transition that occurs at zero temperature. More generally, it's probably easiest to answer your question by comparing quantum criticality to its classical counterpart. At a continuous phase transition the inverse order parameter susceptibility vanishes so that the order parameter correlation function becomes scale-invariant. This means that it decays with distance and time not exponentially but rather gradually in a power-law form. The thermodynamic variables depend only on scale-invariant correlation functions in space for classical criticality, but crucially on both space and time for quantum criticality. This leads to new critical exponents that are quantum in nature depending on details of the order parameter dynamics.

In ferroelectrics classical criticality is difficult to observe in practice. Why isn't the same true for quantum criticality?

As you suggest, the criteria for observing classical and quantum criticality are quite 
different. For example classical criticality just below $T_{c}$ is defined as the region near a finite temperature phase transition where fluctuations in the order parameter are comparable to the average of the order parameter itself. Empirically it has been found that mean-field theory works very well near classical ferroelectric phase transitions, though of course most are first-order. Actually many ferroelectrics reside close to tricritical points at ambient pressure. Therefore it's not surprising that pressure-tuned ferroelectric transitions are continuous, at least in practice. More generally, continuous ferroelectric quantum phase transitions are expected if one is willing to tune not only pressure or composition but also the electric field. As an aside, we should also note that textured states are known to reside near first-order quantum phase transitions, so they can be very interesting too.

\section{What defines the quantum critical region?}

It's important to realize that temperature is not a simple tuning parameter at a quantum phase transition. Indeed temperature provides the low-energy cutoff for quantum fluctuations where the associated time-scale is defined through the Heisenberg

uncertainty relation $\Delta t \sim \frac{\hbar}{k_{B} T}$. In this sense temperature plays the role of a finite-size effect in time at a quantum critical point. The quantum critical region is defined by the interplay between the scale-invariant order parameter fluctuations and the temporal boundary conditions imposed by finite temperature; most importantly it is accessible experimentally with distinct observable signatures.

\section{Now can you please explain why $d+1$ is the effective dimension?}

In the case of purely classical fluctuations, the amplitude for each mode of wavevector $q$ depends only on the temperature and not on its dynamical properties, as we've already noted. Therefore its statistical mechanical description involves only the $d$ dimensions of wavevector (or of real) space. However when quantum fluctuations are present, the mode frequency as well as the temperature are important for the statistical mechanical characterization; for example see the expression for the variance in Figure 1. In general there is a distribution of frequencies $\omega$ associated with each mode that reduces to a $\delta$-function in the special case of a simple harmonic oscillator where $\omega=\Omega$. More generally each mode has a power spectrum distribution of frequencies that results in a statistical mechanical description involving not only the sum over wavevectors but also over frequency $\omega$. The effective number of dimensions to be associated with the dynamics is dependent on the frequency-wavevector dispersion relation. If the dispersion is linear, as it is for ferroelectrics, space and time enter the statistical description on equal footing leading to an overall effective dimensionality of " $d+1$ " referring to $d$ space and 1 time dimensions. Another subtlety is that the effective time dimension is of finite size except in the limit $T \rightarrow 0$ as we've just discussed.

New functionalities are of great interest to the ferroelectrics community, so are there useful low-temperature applications for these materials that could 
be pursued in parallel to studies of quantum criticality?

The trends for future devices are faster, lighter and smaller. Ferroelectric films are used as both active and passive memory elements where data is stored as the presence (or absence) of charge. Reduced operating temperatures lead to lower leakage currents and to increased breakdown fields, both crucial for keeping competitive with faster access and high-density needs.

Electrocaloric cooling, the change in temperature with applied electric field, could be developed to access cryogenic temperatures just as its magnetic counterpart, magnetocaloric cooling, is often used to access millikelvin temperatures and below.

There was some work exploring cryogenic electrocaloric cooling some time ago that was not pursued as the observed effects were too small for practical use...what has changed since then to make you optimistic about this application?

In a nutshell, current thin-film and multicapacitor technologies means that we can increase breakdown fields, particularly at low temperatures without loss of effective volume. It is certainly much easier and cheaper to apply electric rather than magnetic fields, and we'll have more to say about electrocaloric cooling shortly.

We should also note that the radiation-hardness of ferroelectric memories makes them ideal for satellite applications where there is repeated passage through the Van Allen belts and naturally cold temperatures! Indeed in efforts to develop radiation-tolerant electronics, NASA has performed on-orbit tests of ferroelectric random access memories, FRAMs, on micro-satellites (see Figure 3). Furthermore NPSAT1, a small satellite built by the Naval Postgraduate School with FRAMs on board, is due to launch on the SpaceX Falcon Heavy sometime in 2017.

Another potential application for cryogenic ferroelectrics is in phased array radar that would replace large, heavy radar antennae that mechanically rotate. Beam steering would be achieved electrically by varying the phase of a voltage train with a field-tuned LC circuit. In order for such array radar devices to be competitive with their mechanical analogues the dielectric loss must be very low, about $0.01 \%$, and thus this should be a niche for cryogenic ferroelectrics. We should point out that the entire device would not have to be at low temperatures...on-chip electrocaloric cooling for the capacitor could do the job nicely!

So it sounds like there are several low-temperature applications for ferroelectrics that can be explored. Now back to a more general question. What from our knowledge of magnetism can be transferred to ferroelectricity?

There are indeed similarities between ferroelectrics and ferromagnets, but there are also key differences. For example, the polarization is a classical object and thus is not 


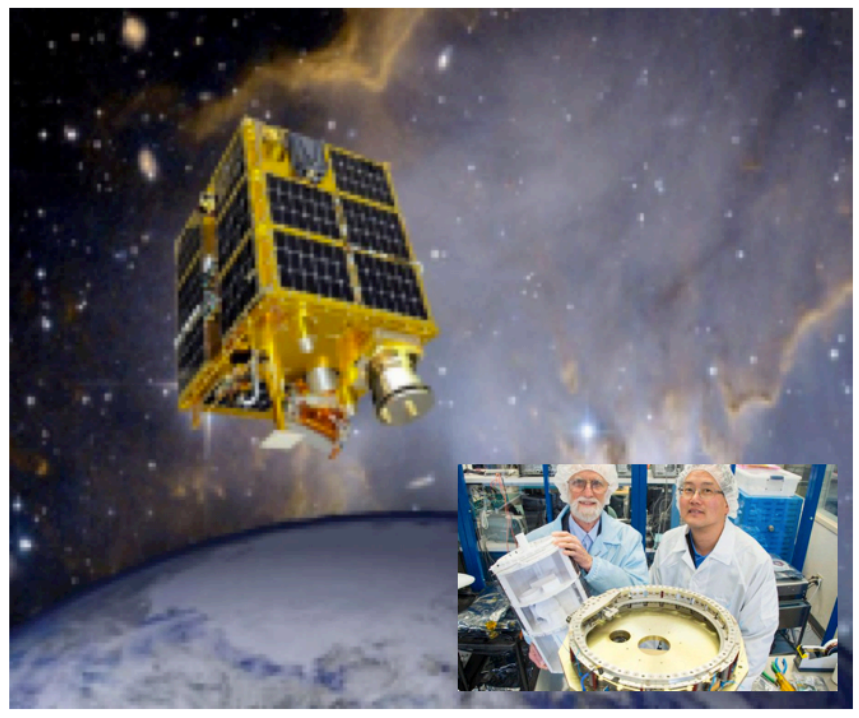

Figure 3. (Left) Artist's rendition of NASA's Fast and Affordable Science and Technology Satellite (FASTSAT) with ferroelectric randon access memory (FRAM) for radiation robustness reprinted from MacLeod et al. [2, 3] with permission and thanks to T.C. MacLeod; (Inset) Naval Postgraduate School scientists R. Panholzer and D. Sakoda with several structural pieces of Naval Postgraduate School Satellite 1 with FRAM [4] due to launch on a STP-2 mission in 2017 on a SpaceX Falcon Heavy rocket [5] (US Navy Photo by Javier Chagoya, reprinted from [4] with permission and thanks to J. Chagoya and the NPS Public Affairs Office).

quantized in contrast to the spin in a magnet. Crystal fields lead to strong anisotropy in ferroelectrics whereas magnetic anisotropy is usually orders of magnitude smaller and is principally due to spin-orbit coupling; this leads to different domain structures in these two distinct classes of materials. The dynamics in ferroelectrics are dominated by propagating vibrational modes, whereas in magnets there is spin precession. These are just some of the reasons one has to be careful going back and forth between magnetism and ferroelectricity, and we'll be discussing this in more detail shortly.

Most of our experiments in quantum criticality are on metallic systems and most ferroelectrics are insulating. So where is the common ground?

We usually emphasize the fact that ferroelectrics are analogous to ferromagnetic insulators. However in the present context, they have interesting features in common with itinerant magnets. In a ferroelectric at high temperatures, the polarization is not well-defined due to dynamical fluctuations in the separation between charges. Similarly in an itinerant magnet, the magnetic moment is not well-defined at high temperatures since the number of electrons in a unit cell is constantly fluctuating. So in that sense the two are not that different. We should add that there also have been studies of doped bulk strontium titanate that indicate very interesting metallic and superconducting 
behaviors. Indeed doped strontium titanate is the superconductor with one of the lowest carrier densities known to date. Its Fermi temperature is lower than its Debye temperature, a feature also seen in many heavy fermion superconductors. Thus it most probably cannot be described by a conventional theory of superconductivity.

\section{So, given our discussion, what can ferroelectricity bring to the study of quantum criticality?}

Empiricially the sensitivities of the ferroelectric transition temperatures to pressure are remarkable! As an example, in order to cover $300 \mathrm{~K}$ changes in magnetic $T_{c}$ 's, we must usually apply hundreds of kilobars, whereas in ferroelectrics the same temperature range can be achieved with more than a factor of ten less in pressure. Furthermore the electric field as another tuning parameter offers tremendous advantages over its magnetic counterpart, as an electric field is significantly easier to apply and doesn't require a lot of extra coils, special cells etc. Also, through gated control of carriers, there is another type of continuous fine-tuning available without the need for multiple samples at different doping levels. In the quantum regime, as we discussed earlier, a system's thermodynamic behavior involves both space and time and hence dynamics; since the dynamics of ferroelectrics and ferromagnets are different, their quantum critical behavior will also be distinct. More generally, another class of materials for experiment is crucial as we collectively explore the possibility of universality in quantum critical phenomena.

$* * * * * * * * * * * * * * * * * * * * * * * * * * * * * * * *$

So we see, there is quite a lot to discuss! We note that there has been tremendous "historical entanglement" here between the fields of ferroelectrics and criticality; the first logarithmic corrections to mean-field exponents due to fluctuations at marginal dimensionality were calculated for a uniaxial ferroelectric [6]. Similarly the transversefield Ising model, one of the simplest models demonstrating a quantum phase transition, was first developed to describe a transition transition in the ferroelectric potassium dihydrogen phosphate $\mathrm{KH}_{2} \mathrm{PO}_{4}$ (often denoted as KDP) [7]. Indeed historically there have been several "waves" of interest in low-temperature paraelectrics that are not completely chronologically distinct; here, in the interest of compactness, we refer the interested reader to previous reviews to discuss these developments [8, 9]. In the 1950s, perovskites like $\mathrm{SrTiO}_{3}$ and $\mathrm{KTaO}_{3}$ were of experimental interest since their dielectric properties were so different from those of (ferroelectric) $\mathrm{BaTiO}_{3}$. Next, in the late 60 s through the mid-80s, with the development of renormalization group, they were settings to test lattice model calculations of quantum critical exponents and to study the importance of long-range dipolar interactions in different dimensions. More recently there has been tremendous interest in the interplay of polarization with other degrees of freedom, so there has been much effort towards modelling phase diagrams of materials for a wide range of temperatures with the aim of raising interesting low-temperature 
phases to room temperature for appropriate applications [10]. A closely related field is that of ferroelastics, the mechanical analogue of ferroelectricity and ferromagnetism, that is associated with shape memory effects [11].

In this article, we'd like to encourage yet another "wave" of interest in the low temperature behavior of paraelectrics/ferroelectrics, one motivated by the quest to discover new quantum states of matter near quantum phase transitions [12, 13, 14, 15]. Materials near their displacive ferroelectric quantum transitions are particularly elegant examples of quantum criticality [16, 17, 18, 19, 20, 21, 22] with few degrees of freedom and propagating dynamics that distinguish them from their magnetic counterparts. Furthermore, as we'll discuss, they are dimensionally tunable so they can be studied experimentally and theoretically at, above and below their upper critical dimensions. Additional degrees of freedom like spin and charge can be added and characterized systematically in these materials, leading to rich phase behavior as yet mostly unexplored.

Let's not get ahead of ourselves. To ensure that everyone is roughly on the same page, we aim for a self-contained article with many references. We apologize in advance to any researchers whose work has been inadvertently overlooked, and we hope that our bibliography will give the interested reader a good starting point to explore topics of interest in more depth. We begin with "Quantum Criticality Basics" in Section II and then continue in III to "Ferroelectrics Necessities." Then (IV) we discuss the specific case of the material $\mathrm{SrTiO}_{3}$ and its behavior at low temperatures. "A Flavor for Low Temperature Applications" is the next section (V) and we end (VI) with several open questions for future research.

\section{Quantum Criticality Basics}

Our aim here is to present key ideas of quantum criticality with minimal formalism to those new to the field, using familiar concepts whenever possible; naturally we refer the reader eager for more detail to a number of excellent reviews [12, 13, 14, 15, 23, 24, 25] In particular our focus will be the temperature behavior of observable quantities near a quantum critical point, eventually associated with ferroelectricity; this goal will guide our discussion. We are all familiar with classical phase transitions where the order parameter develops at a characteristic critical temperature. This standard picture assumes purely classical (thermal) fluctuations which is certainly appropriate for the temperatures of general interest. As we've just discussed in the Introduction, quantum fluctuations also contribute to order parameter fluctuations of modes with characteristic frequencies of the order of or greater than the temperature; here for presentational simplicity we have set the constants $\hbar=k_{B}=1$. However if, as $T \rightarrow 0$, the fluctuationselection of different ground states is enhanced by another tuning parameter, $g$, then there is the possibility of a $T=0$ continous quantum phase transition. 
Let's resume our previous discussion of order parameter fluctuations where we treated each Fourier mode as a simple harmonic oscillator of amplitude $x$ with frequency $\Omega$. The total variance in the mode amplitude is then

$$
\left\langle x^{2}\right\rangle=\left\{n_{\Omega}+\frac{1}{2}\right\} \Omega \chi
$$

where $n_{\Omega}$ refers to the Bose function and $\chi=\frac{1}{\mathcal{K}}\left(=\operatorname{Re} \chi_{\omega=0}\right)$ where $\mathcal{K}$ is the relevant spring stiffness or elastic constant. We recall that for a simple harmonic oscillator

$$
\operatorname{Im} \chi_{\omega}=\frac{\pi}{2} \omega \chi \delta(\omega-\Omega) \quad(\omega>0)
$$

so that we can rewrite (1) as

$$
\left\langle x^{2}\right\rangle=\frac{2}{\pi} \int_{0}^{\infty} d \omega\left\{n_{\omega}+\frac{1}{2}\right\} \operatorname{Im} \chi_{\omega} .
$$

We note that this link between the variance of amplitude fluctuations and the imaginary part of the response, here derived for a simple harmonic oscillator, is actually a much more general result associated with the fluctuation-dissipation (Nyquist) theorem [26].

We can generalize (3) to a sum over all modes labelled by wavevector $q$, for example, in the entire Brillouin zone. Let us now transition to the amplitude of the scalar order parameter $\phi$ that here is a (dipole) moment density that can be either magnetic or electric; we use this terminology for simplicity to avoid confusion with other common symbols often associated with pressure. Then, following our previous argument, the variance of the amplitude fluctuations of the moment is

$$
\left\langle\delta \phi^{2}\right\rangle=\frac{2}{\pi} \sum_{q} \int_{0}^{\infty} d \omega\left\{n_{\omega}+\frac{1}{2}\right\} \quad \operatorname{Im} \chi_{q \omega}
$$

where $\phi=\bar{\phi}+\delta \phi, \bar{\phi}$ is the average, $\langle\delta \phi\rangle=0$ and

$$
\operatorname{Im} \chi_{q \omega}=\frac{\pi}{2} \omega \chi_{q} \delta\left(\omega-\omega_{q}\right) \quad(\omega>0)
$$

in the propagating limit where $\omega_{q}$ is the oscillator frequency of the mode of wavevector $q$; naturally more general power spectra are also possible [27].

Equation (4) is composed of a strongly temperature-dependent contribution $\left\langle\delta \phi_{T}^{2}\right\rangle$ involving the Bose factor $n_{\omega}$; the remainder $\left(\left\langle\delta \phi_{Z P}^{2}\right\rangle\right.$ involving the factor $\frac{1}{2}$ instead of $\left.n_{\omega}\right)$ is due to "zero-point" fluctuations. Here we focus on $\left\langle\delta \phi_{T}^{2}\right\rangle$ since it is dominant in determining the temperature-dependence of the observable properties of interest here. We note that the zero-point contribution mainly affects the $T=0$ properties and as noted previously can drive a quantum phase transition; in particular here it is assumed just to renormalize the underlying parameters of the free energy-energy expansion in the vicinity of the zero-temperature transition [26] that we'll present shortly. Let us now return to equation (4). At high temperatures $(T>>\omega), n_{\omega} \approx \frac{T}{\omega}$; invoking causality in the form of the Kramers-Kronig relations, we obtain a generalized equipartition theorem $[26]$

$$
\left\langle\delta \phi_{T}^{2}\right\rangle \approx T \sum_{q<q_{B Z}} \chi_{q} \quad\left(T>>\omega_{q} \text { for } q<q_{B Z}\right) .
$$


Here we see that the dynamics drop out completely of the classical equilibrium description. We also note that in (6) we have a d-dimensional wavevector summation over the Brillouin zone that implies a d-dimensional theory in real space.

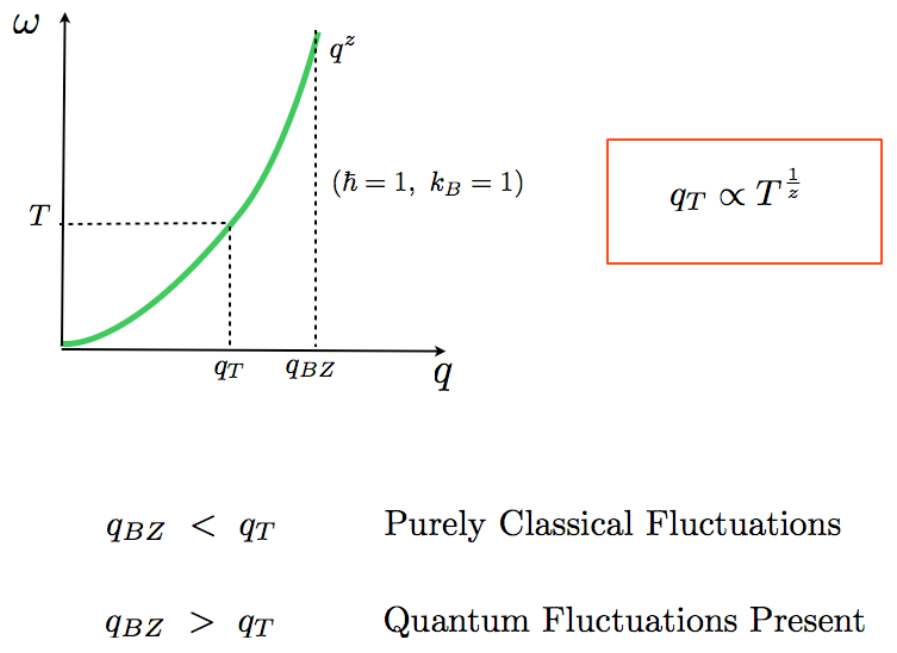

Figure 4. Important wavevectors and the dispersion $\omega \propto q^{z}$.

By contrast, in the regime $(T<<\omega)$ where quantum effects are important, $n_{\omega} \approx e^{-\frac{\omega}{T}}$ and the dynamics remain. In order to proceed with our treatment of (4), we therefore must consider the dispersion $\omega_{q}$; please see Figure 4. In particular we'll get a purely classical result, (6), if all the modes in the Brillouin zone are excited; otherwise the modes will be classical up to a wavevector cutoff determined by quantum mechanics (see Figure 4). The relevant wavevector scales are the Brillouin zone $\left(q_{B Z}\right)$ and the thermal $\left(q_{T}\right)$ wavevectors, where the latter's temperature-dependence, via the dispersion $\omega_{q} \propto q^{z}$ for low $q$, is

$$
q_{T} \propto T^{\frac{1}{z}}
$$

and we note that $\frac{1}{q_{T}}$ is a generalized deBroglie wavelength that correponds to the usual free-particle case when $z=2$. We emphasize that the smaller of the two wavevector scales $q_{T}$ and $q_{B Z}$ serves as a cutoff for the classical fluctuations. If $q_{T}<q_{B Z}$ then not all modes in the Brillouin zone are thermally excited; in this case the dynamical exponent enters (4) via $q_{T}$ and thus quantum effects contribute to the variance of the order parameter fluctuations.

Let us now apply these ideas towards analyzing (4) when the important cutoff is $q_{T}$. We revisit the most strongly temperature-dependant part of the $\omega$-integral in (4), breaking it up into two separate parts as approximately

$$
\mathcal{I}=\mathcal{I}_{1}+\mathcal{I}_{2} \approx \int_{0}^{T} d \omega\left(\frac{T}{\omega}\right) \operatorname{Im} \chi_{q \omega}+\int_{T}^{\infty} d \omega e^{-\frac{\omega}{T}} \operatorname{Im} \chi_{q \omega} .
$$

We note that for $q<q_{T}$ the delta function in (4) and (5) ensures that only $\mathcal{I}_{1}$ contributes in (8); for $q>q_{T}, \mathcal{I}_{1}$ is zero and $\mathcal{I}_{2}$ involves an exponential damping factor and thus can 
be ignored to leading order. Therefore, using Kramer-Kronig relations, we can write (4) as

$$
\left\langle\delta \phi_{T}^{2}\right\rangle \approx T \sum_{q<q_{T}} \chi_{q} \quad\left(T<<\omega_{q} \quad \text { for } q<q_{T}\right) .
$$

where the dynamics are present via (7). In this approach, the key distinction between the two moment variances, (6) and (9), lies in their wavevector cutoffs: in the purely classical case $(6)$ it is a constant $\left(q_{B Z}\right)$, whereas when quantum effects are important, (9), the dynamical exponent $z$ enters through $q_{T}$.

Using the Landau theory of phase transitions (also called the Landau-Devonshire theory in the area of ferroelectric phase transitions) [26, 28, 29] combined with (6) and (9), we can relate the variance $\left\langle\delta \phi_{T}^{2}\right\rangle$ to the susceptibility $\chi$, an observable quantity $[22,30]$. In the magnetic and ferroelectric cases of interest here,

$$
\chi_{q}^{-1} \propto \kappa^{2}+q^{2}
$$

where $\kappa$ is the inverse correlation length so that in the limit of $q \rightarrow 0$ we have

$$
\chi^{-1} \propto \kappa^{2} .
$$

We recall that Landau theory is a symmetry-based description of macroscopic properties near a phase transition; here we will be considering behavior on length-scales greater or equal to $\frac{1}{q_{T}}$. This coarse-graining ensures that the main effects of zero-point fluctuations are absorbed in the Landau coefficients but that thermal effects show up through the fluctuations of the order parameter field coarse-grained over $\frac{1}{q_{T}}$. We assume that this scale is large enough so that a Taylor expansion of the free energy is still reasonable for our applications.

The Landau free energy density for a system with moment $\phi$ and conjugate field $\mathcal{E}$ is

$$
f=\frac{1}{2} \alpha \phi^{2}+\frac{1}{4} \beta \phi^{4}+\frac{1}{2} \gamma|\nabla \phi|^{2}-\mathcal{E} \phi
$$

where $\alpha \rightarrow 0$ at the transition and $\beta$ and $\gamma$ are positive constants for a continuous phase transition to a uniformly ordered state that we wish to consider. Minimizing this free energy with respect to the order parameter $\phi$, we obtain

$$
\mathcal{E}=\alpha \phi+\beta \phi^{3}-\gamma \nabla^{2} \phi
$$

Solving for $\phi$ in (13), we obtain its most probable value associated with the maximum of its probability distribution. In order to determine the observed moment, we consider the effects of fluctuations due to a random (Langevin) field added to $\mathcal{E}$. More specifically we must average over the random fluctuations in (13) using $\phi \rightarrow \bar{\phi}+\delta \phi$ where $\bar{\phi}$ is the average and $\langle\delta \phi\rangle=0$; we obtain

$$
\mathcal{E}=\left(\alpha+3 \beta\left\langle\delta \phi^{2}\right\rangle\right) \bar{\phi}+\gamma \nabla^{2} \bar{\phi}
$$

to lowest order where we note that the variance term arises from the anharmonic effects of the cubic term in the equation of state. In the limit of small $\bar{\phi}$ and $\mathcal{E}$, we can Fourier transform this expression to obtain

$$
\chi_{q}^{-1}=\left(\alpha+3 \beta\left\langle\delta \phi^{2}\right\rangle\right)+q^{2} .
$$


Taking the expression (15) in the $q \rightarrow 0$ limit and again retaining the most strongly temperature-dependent terms, we find that

$$
\lim _{T \rightarrow 0} \kappa^{2} \propto\left\langle\delta \phi_{T}^{2}\right\rangle
$$

where we have assumed a quantum critical point (QCP) so that $\alpha \rightarrow 0$ as $T \rightarrow 0$.

The careful reader may ask why we are distinguishing between the most probable and the average (observed) value of $\phi$, and this question can be addressed by discussion of equation (15). If the coarse-graining underlying our Landau theory is macroscopic, then the $q$ phase space and thus the variance is small, except in the Ginzburg regime to be defined below, so that the the most probable and the average values are essentially identical. However, as we have already noted, our coarse-graining is mesoscopic and not macroscopic and therefore we must include the variance in our calculations. An alternative way to address this issue is to recall that the true equation of state is found by averaging over the most probable one ([31]); for a Gaussian theory of course the average and the most probable values of $\phi$ are identical. Finally we emphasize that (16) is only valid near a $T_{c}=0$ phase transition since for a nonzero $T_{c}$ there are additional terms proportional to $T_{c} \neq 0$ so that this expression of proportionality no longer holds [30].

We can now combine (9), (10) and (16) to determine the temperature-dependence of the susceptibility near a quantum critical point; towards this goal, we write

$$
\kappa^{2} \propto \sum_{q<q_{T}} \frac{T}{\kappa^{2}+q^{2}} \approx T \int_{\kappa}^{q_{T}} \frac{q^{d-1}}{q^{2}} \approx T q_{T}^{d-2}\left\{1-\left(\frac{\kappa}{q_{T}}\right)^{d-2}\right\} .
$$

where, using $q_{T} \propto T^{\frac{1}{z}}$, we are tempted to neglect the $\frac{\kappa}{q_{T}}$ term on the right-hand side of (17) and write

$$
\chi^{-1} \propto \kappa^{2} \propto T^{\frac{(d+z-2)}{z} .}
$$

(18) shows that the quantum critical exponent for the susceptibility is $\frac{d+z-2}{z}$ that can be compared to the classical value of unity (e.g. the Curie susceptibility) outside the Ginzburg regime. Now we can ask, when is this approach valid? We can answer this question by rearranging (17) to yield

$$
\left(\frac{\kappa}{q_{T}}\right)^{2} \propto T^{\frac{(d+z-4)}{z}}\left\{1-\left(\frac{\kappa}{q_{T}}\right)^{d-2}\right\} .
$$

From (19), we see that $\left(\frac{\kappa}{q_{T}}\right) \rightarrow 0$ as $T \rightarrow 0$ if $d_{e f f} \equiv d+z>4$; in this case the inverse susceptibility in the approach to a QCP has the temperature-dependence displayed in (18) and no further fluctuation effects need to be considered. $d_{\text {space }}^{\text {upper }}=4-z$ is thus the upper critical spatial dimension of this theory. An analogous treatment leads to $d^{\text {upper }}=4$ for the purely classical description [13,30]; it is more complicated than the $T \rightarrow 0$ case due to the presence of more finite terms, so here we will simply state the result. 
Let us now return to (17) and (19) with cutoff $q_{T}$. It is as if the frequency (or time) dimension is equivalent to $z$ wavevector (or space) dimensions through the dispersion relation that relates frequency to $z$ factors of wavevector $\left(\omega \propto q^{z}\right)$. Perhaps it is easier to state that the inclusion of dynamics in quantum critical phenemona theory reduces the upper critical dimension from 4 in the classical limit (where dynamics can be ignored) to $4-z$ (where dynamics must be considered). From this standpoint, we are usually above the upper critical dimension at a quantum phase transition whereas we are below it for its classical counterpart.

We have already noted that the frequency dimension is truncated by the Bose function and can be envisioned to have a finite-size of order $T$, so that the corresponding time dimension is of finite-size of order $\frac{1}{T}$. The crucial point here is that the role of temperature near a quantum critical point is to constrain the temporal dimension; for $d<d_{\text {space }}^{\text {upper }}=4-z$, thermal effects can be treated compactly via the ideas of finite-size scaling. More generally, we note that the frequency integration in (4) can be performed by contour integration where the poles for the Bose function are imaginary [27]. This is an alternative to the real-frequency and real-time description given here, and it yields the same results mathematically.

\section{Quantum Criticality: Key Concepts}

- The dynamical properties of the order-parameter fluctuations affect the equilibrium thermodynamic properties in the quantum critical regime (in contrast to their classical counterparts where only thermodynamic properties usually only depend on statics).

- The dynamical exponent $z$, defined by the dispersion relation $\left(\omega \propto q^{z}\right)$ at the quantum critical point, plays an important role in quantum critical phenomena.

- The effective dimensionality, $d_{e f f}=d+z$, is the sum of the spatial and temporal dimensions, where the latter is represented by the dynamical exponent.

- Near a quantum critical point (QCP), temperature acts as a boundary condition on time and not as a simple tuning parameter.

- There exists a finite-temperature quantum critical region near a QCP where there is a gapless dispersion, $q_{T}<<q_{B Z}$ and $q_{T} \propto T^{\frac{1}{z}}$.

- At sufficiently low temperatures near a QCP, the temperaturedependence of the inverse susceptibility is

$$
\chi^{-1} \propto T^{\frac{d+z-2}{z}} \quad(d+z>4)
$$

(with weak logarithmic corrections for $d+z=4$ ) 


\section{Ferroelectrics Necessities}

So why study the influence of quantum effects in materials with ferroelectric tendencies? Before addressing this question, let us familiarize ourselves with key features of ferroelectrics (FE); here we emphasize aspects important to our topic at hand, referring the reader eager for further information to several detailed reviews and books $[9,28,32,33,34,35,36,37,38,39]$.

From a working "engineering" standpoint, a ferroelectric is a material that has a spontaneous polarization that is switchable by an electric field of practical magnitude; in a finite system the polarization is defined as the dipole moment per volume averaged over the unit cell volume [37]. In Figure 5, the link between ferroelectrics, pyroelectrics, piezoelectrics and dielectrics is presented graphically. In piezoelectrics an applied mechanical stress results in a voltage and vice versa $[28,32,33,36]$. A change in temperature causes an electrical polarization in a pyroelectric $[28,32,33,36]$ and it is the practical switchability of this polarization that distinguishes a pyroelectric from a ferroelectric [36]. Inversion but not time-reversal symmetry is broken at a ferroelectric transition. The development of a spontaneous polarization results from electric dipoles that are classical and non-relativistic; they are spatially extended within the unit cell. A ferroelectric displays a polarization-electric field hysteresis that is analogous to the magnetization-magnetic field hysteresis measured in magnetic materials. Because the polarization is the electric dipole moment per unit volume it has the units of charge/area [28]. Only the relative polarization, not its absolute value, is measured and this is usually performed by integrating a switching current [37].

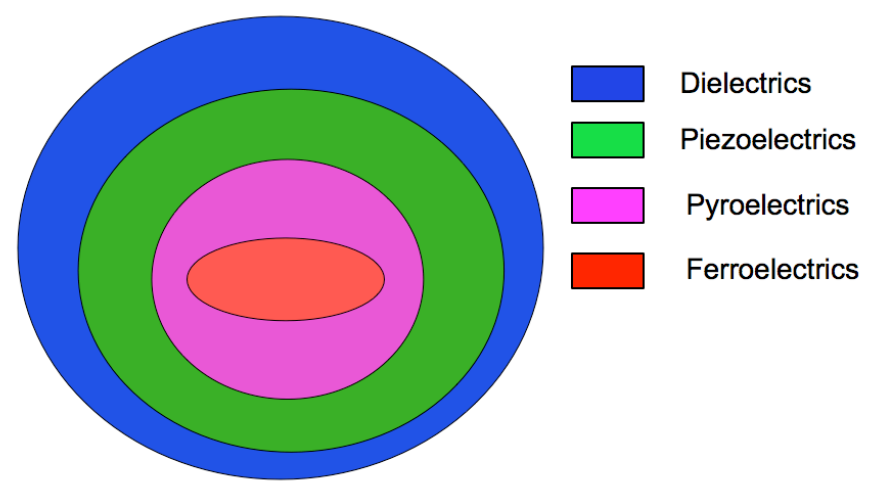

Figure 5. Venn diagram indicating graphically the relationship beween ferroelectrics, pyroelectrics, piezoelectrics and dielectrics. Applied stress and temperature changes lead to electrical polarization in piezoelectrics and in pyroelectrics respectively $[28,32,33,36]$; the switchability of this polarization in a field of practical magnitude (and is less than the breakdown electric field) is what distinguishes a ferroelectric from a pyroelectric [36].

Qualitively there are two types of ferroelectric transitions [28]: those driven mainly by amplitude fluctuations (displacive) and those driven mainly by angular fluctuations 
(order-disorder) at atomic scales. In the latter case, the entropy change at the transition is higher than in the former situation. At low temperatures, particularly as $T \rightarrow 0$, ferroelectic transitions are predominantly displacive and we'll return to this topic when we discuss analogies with itinerant magnetism in the next section. Here we are implicitly discussing ionic ferroelectricity where the polarization results from ionic displacements, though we do note "electronic ferroelectricity" in molecular crystals where the polarization is due to the ordering of electrons [40]. We emphasize that ionic ferroelectrics can be order-disorder and/or displacive in their character. In these ferroelectics, strong coupling of the polarization and the lattice often leads to first-order transitions, both of order-disorder and displacive varieties.

In conventional (ionic) ferroelectrics, the electric dipoles associated with the spontaneous polarization are produced by atomic rearrangements and they develop longrange order at a ferroelectric transition. Indeed the soft-mode theory of ferroelectricity [28, 41, 42, 43], a lattice dynamics description, links the diverging dielectric response with a vanishing phonon frequency and can indeed be viewed as an early model of quantum criticality. We can glean a flavor for the soft-mode approach by considering the frequency-dependent electrical permittivity, $\epsilon_{\omega}$ of a simple diatomic harmonic lattice

$$
\epsilon_{\omega}=\epsilon_{\infty}+\frac{\epsilon_{0}-\epsilon_{\infty}}{1-\frac{\omega^{2}}{\omega_{T O}^{2}}}
$$

where $\epsilon_{0}$ and $\epsilon_{\infty}$ refer to the permittivities at zero (static) and infinite frequencies respectively. In the absence of free charge, the zero and the pole of $\epsilon_{\omega}$, respectively, determine the longitudinal and transverse optical mode frequencies $\omega_{L O}$ and $\omega_{T O}$ resulting in the relation $[34,44]$

$$
\frac{\epsilon_{0}}{\epsilon_{\infty}}=\left(\frac{\omega_{L O}}{\omega_{T O}}\right)^{2}
$$

that links the softening of a polar (transverse optical) phonon to the development of ferroelectricity.

This minimalist approach to soft-mode theory can of course be generalized to include anharmonicities and many polar modes where the frequencies are either measured [43] or calculated using first-principles methods [45, 46, 47]. We emphasize that a finite spontaneous polarization can only exist in a crystal with a polar space group [45], though this does not ensure its switchability in a practical electrical field. A structural signature of ionic ferroelectricity is that the finite polarization crystalline configurations result from small polar distortions of a high-symmetry (paraelectric) structure so that there is a simple pathway between them [45]. In Figure 5 we display the crystal structure of the well-studied perovskite ferroelectric $\mathrm{BaTiO}_{3}$, its paraelectric (cubic) structure and two of its polarization states. From a first-principles perspective, a fingerprint of ferroelectricity is the presence of unstable polar phonons in high-symmetry reference structures and this has been a successful method for characterizing known and new ferroelectric materials [45]. Until relatively recently, it has been tacitly assumed 
that the polar phonon frequency vanishes as a function of temperature but of course other tuning parameters (like pressure) could achieve this softening as well.

a)

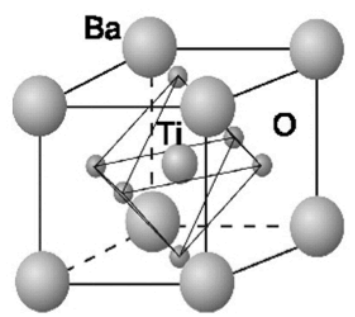

Cubic phase b)

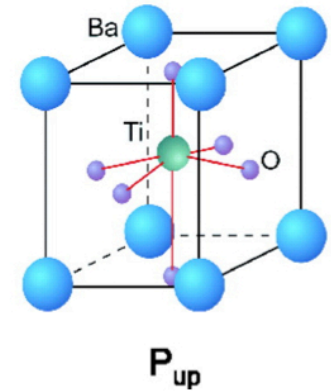

c)

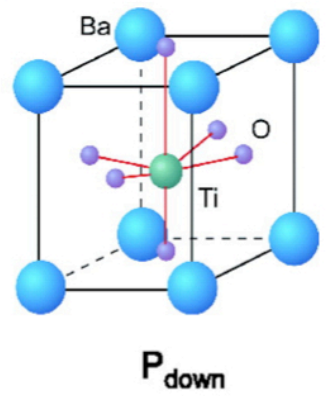

Figure 6. Crystal structures of the perovskite ferroelectric $\mathrm{BaTiO}_{3}$. a) Hightemperature cubic paraelectric and room-temperature tetragonal ferroelectric structures for (b) up and (c) down polarizations respectively $\left(P_{u p}\right.$ and $\left.P_{\text {down }}\right)$ indicating the relative displacements of the positively charged Ti and negatively charged O ions; reprinted from Ahn et al. [48] with permission.

It is worth comparing the relative strengths of the electric and magnetic dipole forces. In atomic units $F_{M}$, the force between two magnetic dipoles at a distance $r$, is

$$
F_{M}=\frac{\mu_{0} \mu_{B}}{4 \pi r^{3}} \equiv \frac{\alpha_{F}^{2}}{4 \pi}\left(\frac{a_{B}}{r}\right)^{3}
$$

where $a_{B}=0.05 \mathrm{~nm}$ and $\alpha_{F} \equiv \frac{1}{137}$ are the Bohr magneton and the fine structure constant respectively; by contrast, for an electric dipole $p=e \Delta a_{B}$, the dipolar interaction force is

$$
F_{D}=\frac{p^{2}}{8 \pi \epsilon_{0} r^{3}} \equiv \frac{\Delta^{2}}{4 \pi}\left(\frac{a_{B}}{r}\right)^{3},
$$

where the parameter $\Delta=O(1)$ is determined by effective charges and atomic displacements [49]. The ratio of the ferroelectric to ferromagnetic dipolar forces is then of order $\left(\frac{\Delta}{\alpha}\right)^{2} \equiv(137)^{2}$, indicating that long-range interactions are more significant in ferroelectrics than in generic magnetic systems. This ratio is a contributing factor towards explaining why the Ginzburg regime, where long-wavelength ("infrared") fluctuations govern the critical behavior, in ferroelectrics is empirically significantly smaller than its counterpart in magnets in many cases [29]; classically the Ginzburg regime below $T_{c}$ is defined by the temperature interval close to a phase transition where order parameter fluctuations are comparable or larger than the average value of the order parameter itself. However corrections to simple mean-field (Landau) theory due to anisotropic dipolar forces and anisotropic elastic interactions may be important. For example, the first logarithmic corrections to mean-field exponents due to fluctuations at marginal dimensionality were calculated for a three-dimensional uniaxial ferroelectric $[6,50,51]$; these predictions were confirmed by experiment $[52,53]$ and played an 
important role in the development of the renormalization group approach to classical phase transitions $[54,55]$.

In the previous section we related $\left\langle\delta \phi_{T}^{2}\right\rangle$ to $\chi(T)$ using (6), (9) and (16); let's now apply these results to $d=3$ ferroelectrics where we are considering a QCP where the gap in the polar optical mode vanishes with a resulting dispersion as $\omega \propto q$ as measured by neutron scattering $[56,57,58]$ so the dynamical exponent $z=1$. In the proximity of a transition where $\alpha=0$, we have at long wavelengths $(q \rightarrow 0)$

$$
\chi(T)^{-1} \approx T \int_{\kappa}^{q_{c}} \frac{q^{d-1} d q}{q^{2}}
$$

where $q_{c}$ is the cutoff appropriate for the temperatures of interest; here we are implicitly neglecting the temperature-dependence of $\kappa$ which, according to (19), is reasonable for $T \rightarrow 0$ if $d+z>4$. At high temperatures $\left(T \gg \omega_{q}\right.$ for $\left.q<q_{B Z}\right), q_{c}=q_{B Z}$ has no temperature-dependence so we recover the Curie result $\chi^{-1} \propto T$; here we have assumed that $\kappa$ has saturated and thus is constant for these high temperatures. However when quantum effects become important $\left(q_{T}<<q_{B Z}\right), q_{c}=q_{T} \propto T^{1 / z}$; applying the results (17), (18) and (19) to $d=3$ ferroelectrics $(z=1)$, we obtain

$$
\chi^{-1} \propto T^{\frac{d-2+z}{z}}=T^{2}
$$

which we emphasize is distinct from the classical (Curie) behavior $\left(\chi^{-1} \propto T\right)$; since $d_{\text {eff }}=d+z=4$ we also have $\log$ corrections that are usually difficult to observe experimentally. We note that we have reproduced a result first calculated diagrammatically $[16,17,59]$ and then rederived using other methods $[18,19,20,22,60]$.

A critical reader may note that here we have neglected the long-range dipolar interactions discussed previously; several theoretical studies [16, 17, 18, 22] indicate that their main effect near a QCP is to produce a gap in the longitudinal fluctuations, but that the transverse fluctuations remain critical. This conclusion is supported by recent measurements [22] of $\chi(T)$ near a ferroelectric QCP (FE-QCP) indicating good agreement with (25). We should stress that at a QCP with $d+z>4$, both $\kappa$ and $q_{T}$ go to zero; however in this case, as we saw in (19), the ratio $\frac{q_{T}}{\kappa}$ diverges as $T \rightarrow 0$ so it is the "ultraviolet" fluctuations that are crucial. By contrast at a classical transition, $\kappa \rightarrow 0$ and the wavevector cutoff $q_{c}=\min \left\{q_{T}, q_{B Z}\right\}$ remains constant, and if $d<4$ the "infrared" fluctuations are important. The key roles of very different fluctuation regimes at classical and at quantum critical points suggests why the influence of dipolar interactions is distinct in these two cases.

Anisotropic elastic effects in ferroelectris has also been studied [61]. The resulting domains have sufficiently slow dynamics, perhaps due to their physical extent or to pinning, that they do not seem to contribute to low-temperature thermodynamic quantities on measurable time-scales studied to date [22].

Analogous to Einstein's approach to the specific heat problem [34], we can also consider the situation where the low-energy excitations are dispersion-free with a single frequency $\omega_{0}$. This is just the case of a simple harmonic oscillator [27] so we have

$$
\chi(\omega) \propto \frac{\omega_{0}}{\omega^{2}-\omega_{O}^{2}}
$$


and

$$
\chi^{\prime \prime}(\omega) \propto \frac{\delta\left(\omega-\omega_{0}\right)}{\omega_{0}} .
$$

Using the identity for the Bose function

$$
n\left(\frac{\omega}{T}\right)+\frac{1}{2}=\frac{1}{2} \operatorname{coth}\left(\frac{\omega}{2 T}\right),
$$

we input (27) into the general expression for the moment amplitude variance (4) to obtain

$$
\left\langle\delta \phi^{2}\right\rangle \propto \frac{1}{\omega_{0}} \operatorname{coth}\left(\frac{\omega_{0}}{2 T}\right) .
$$

Taking the $q \rightarrow 0$ limit of (15) we obtain

$$
\chi^{-1}=\left(\alpha+3 \beta\left\langle\delta \phi^{2}\right\rangle\right)
$$

where $\alpha$ and $\beta$ are defined in (12); both are finite since we are not at a phase transition. Combining (29) and (30), we then obtain

$$
\chi^{-1}=\left[\alpha+\frac{3 A \beta}{\omega_{0}} \operatorname{coth}\left(\frac{\omega_{0}}{2 T}\right)\right]
$$

which can be rewritten in the Barrett form $[62,63]$

$$
\chi=C\left[\frac{\omega_{0}}{2} \operatorname{coth}\left(\frac{\omega_{0}}{2 T}\right)-T_{0}\right]^{-1}
$$

where $C=\frac{\omega_{0}^{2}}{6 \beta}$ and $T_{0}=-\frac{\alpha A}{6 \beta}$ are constants written in terms of the original parameters. We re-emphasize that the Barrett (or rather "Einstein-Barrett") expression is for dispersion-free excitations [28]; it is thus not valid in the immediate vicinity of a quantum critical point where, similar to the situation in the Debye model [34, 44], excitations of different wavevectors have different frequencies.

The Grüneisen ratio, $\Gamma=\frac{\tilde{\alpha}}{c_{P}}$ where $\tilde{\alpha}$ and $c_{p}$ are the thermal expansion and the specific heat respectively, has been identified as a physical quantity that diverges at a $\mathrm{QCP}$ and is constant at a classical critical point $[64,65,66]$. The Grüneisen ratio is then a useful bulk thermodynamic probe to locate, classify and categorize QCPs in a diverse set of materials, so let's now use the methods we've developed to determine $\Gamma(T)$ near a FE-QCP. As an aside, we note that this Grüneisen ratio is to be distinguished from the Grüneisen parameter that measures the logarithmic change of a particular mode frequency as a function of volume change; the two quantities are only simply related when the lattice frequencies are temperature-independent which is definitely not the case for the (predominantly) displacive ferroelectrics (DFEs) of interest here.

Using Maxwell's relations, the Grüneisen ratio can be written as the effect of a volume change on a solid's total thermal energy, $\Gamma=\frac{1}{V} \frac{\partial V}{\partial U}$. Because $d=3$ displacive quantum paraelectrics $(z=1)$ reside in their marginal dimension $\left(d_{\text {eff }}=\right.$ 4), their critical behavior can be described by a self-consistent mean-field theory where fluctuation corrections due to anharmonicities are included via the fluctuationdissipation theorem; we've already implemented this approach in (15) where the 
Gaussian fluctuations are treated to leading order using (9). This approach is only strictly valid for $d_{\text {eff }}>4$, but the weakly temperature-dependent logarithmic corrections to mean-field theory are likely to be too small to be observable in most experiments [22]. The free energy as a function of the polarization change $\left(\delta \phi_{E}\right.$ where here $\phi_{E}$ is the electric dipole)

$$
F\left(\delta \phi_{E}, \delta V\right)=\frac{\alpha}{2} \delta \phi_{E}^{2}+\frac{a}{2} \delta V^{2}-\eta(\delta V)\left(\delta \phi_{E}^{2}\right)
$$

where on symmetry grounds the form of the coupling term is even in $\delta \phi_{E}$ but odd in $\delta V$, the change in volume from the equilibrium $T=0$ value; $\alpha=0$ at a phase transition and $a$ and $\eta$ are constants.

\begin{tabular}{|c|c|c|}
\hline & $T \rightarrow T_{c}^{+} \quad\left(T_{c}>0\right)$ & $T \rightarrow 0^{+} \quad\left(T_{c}=0\right)$ \\
\hline $\begin{array}{c}\text { Inverse Dielectric } \\
\text { Susceptibility } \\
\chi^{-1}\end{array}$ & $T$ & $T^{2}$ \\
\hline $\begin{array}{c}\text { Grüneisen Ratio } \\
\Gamma=\frac{\alpha}{c_{P}}\end{array}$ & Constant & \\
& C & $T^{-2}$ \\
& Diverging \\
\hline
\end{tabular}

Table I: Expected temperature-dependences of two experimental probes in the approach to $d=3$ ferroelectric critical points we reproduce susceptibility results found elsewhere $[16,17,18,19,20,22,59,60]$. Here $T \rightarrow T_{c}^{+}$and $T \rightarrow 0^{+}$refer to classical and to quantum critical points respectively. In the approach to a classical critical point, the inverse dielectric susceptibility displays Curie $\left(\chi^{-1} \propto T\right)$ behavior; for $T \rightarrow 0^{+}$, it scales as $\chi^{-1} \propto T^{2}$ where here we are neglecting weak logarithmic corrections for the relevant case $d+z=4$. We note that $\chi=\epsilon-1$ where $\epsilon$ is the dielectric function. The Grüneisen ratio, $\Gamma=\frac{\alpha}{c_{P}}$ where $\alpha$ and $c_{p}$ are the thermal expansion and the specific heat respectively, diverges near a quantum critical point $\left(\Gamma \propto T^{-2}\right)$; by contrast it remains constant near a classical one and thus is an important signature of quantum criticality $[64,65,66]$.

Minimizing (33) with respect to volume and, using (9) to average over fluctuations to get the most probable result, we obtain

$$
\langle\delta V\rangle \propto\left\langle\delta \phi_{E}^{2}\right\rangle
$$


so that

$$
\Gamma_{F E}(T)=\frac{1}{V}\left(\frac{\delta V}{\delta U}\right) \propto \frac{\left\langle\delta \phi_{E}^{2}\right\rangle}{\delta U}
$$

Because neither the numerator or the denominator has a singularity in $\left(T-T_{c}\right)$ for a finite transition temperature $T_{c}$, we expect that

$$
\Gamma_{C F E}\left(T \rightarrow T_{c}\right) \propto\left(T-T_{c}\right)^{0}
$$

will be independent of temperature; this is supported by experiment reporting the identical temperature-dependences of thermal expansion and specific heat near finitetemperature ferroelectric phase transitions [28].

However in the approach to a $T \rightarrow 0^{+}$FE-QCP, we can use (16) to write

$$
\lim _{T \rightarrow 0^{+}}\left\langle\delta \phi_{E}^{2}\right\rangle \propto \chi^{-1} \propto T^{2} .
$$

Analogous to the Debye approach to the specific heat [44], the change in energy is equal to the temperature multiplied by the number of accessible modes

$$
\delta U_{Q F E} \propto T\left(q_{T}^{d}\right)
$$

so that the temperature-dependence of $\Gamma$ in the vicinity of a $(d=3)$ FE-QCP is

$$
\Gamma_{Q F E}=\left(\frac{\delta V}{\delta U}\right) \propto\left(\frac{\left\langle\delta \phi_{E}^{2}\right\rangle}{\delta U}\right) \propto \frac{\chi^{-1}}{T q_{T}^{d}}=\frac{T^{2}}{T^{4}}=\frac{1}{T^{2}}
$$

that diverges with decreasing temperature and thus is dramatically different from the temperature-independent classical case (36); here we are implicitly considering the strongly temperature-dependent part of $\phi_{E}$.

Since $\Gamma=\frac{\tilde{\alpha}}{c_{P}}$ where $\tilde{\alpha}$ and $c_{P}$ are the thermal expansion and the specific heat respectively, its experimental determination involves two distinct measurements. Not only does the temperature-dependence of $\Gamma$ signify the importance of quantum fluctuations, but it is also an independent determination [67] of the dynamical exponent $z$. In Table I. we summarize the distinctive temperature-dependences of the inverse susceptibility and the Grüneisen ratio in the vicinity of three-dimensional classical and quantum displacive ferroelectric critical points. 


\section{Ferroelectric Necessities: Key Concepts}

- A ferroelectric has a spontaneous polarization that is switchable by an electric field.

- Inversion symmetry is broken in the ferroelectric phase.

- The temperature-dependence of observable quantities (e.g. susceptbility) in the vicinity of both classical and quantum critical points can be determined using a self-consistent mean-field theory where fluctuation corrections due to anharmonicities are given by the fluctuationdissipation theorem.

- The Barrett form of $\chi(T)$ results if a single Einstein frequency is assumed; this is not valid in the vicinity of a QCP where the wavevectordependence of the excitation spectrum (dispersion) is important.

- The Grüneisen ratio diverges with decreasing temperature near a quantum ferroelectric critical point but remains constant near its classical counterpart.

More generally paraelectrics near displacive ferroelectric quantum critical points offer appealing examples of quantum critical behavior often without the complications of dissipation and damping that occur in metallic magnetic systems. Furthermore because their dispersion is linear $(z=1)$, quantum critical paraelectrics can be studied just below, at or just above their upper critical dimension $\left(d^{\text {upper }}=3+1=4\right)$ making detailed comparison between theory and experiment possible in ways that are not so straightforward for their metallic magnetic counterparts (e.g. $z=3$ for a metallic ferromagnet) $[12,18,22]$. It is thus perhaps not so surprising that some of the earliest theoretical studies of quantum criticality were done in a paraelectric setting $[16,17]$.

A key similarity between displacive ferroelectrics (DFEs) and metallic magnetic systems is that in both material classes amplitude fluctuations of the appropriate moments on length-scales of order their unit cells are significant so that it is relatively straightforward to suppress their orderings to $T \rightarrow 0$. By contrast, in insulating magnets and order-disorder ferroelectrics the moment fluctuations are mainly orientational on length-scales of order their unit cells in the high-temperature phase; it is therefore challenging to prevent ordering at low temperatures for the study of quantum criticality, though there are indeed some magnetic examples [15, 68, 69, 70, 71]. As an aside, we should note that in the literature the descriptives metallic and itinerant are often used interchangeably; here we will use both terms to mean that the volume of the Fermi surface encloses the magnetic carriers. Of course the dynamics in displacive ferroelectrics (propagating vibrational modes) are distinct from those in itinerant magnets (spin precession and dissipative spin dynamics) and this will result in different quantum 
critical behavior. The issue of universality near quantum phase transitions is still one of open discussion, and a new class of materials for detailed study could shed light on this central issue [72]. With this goal in mind, in Table II. we summarize key similarities and differences between displacive ferroelectrics and itinerant ferromagnets, focussing on characteristics most relevant for the study of quantum criticality.

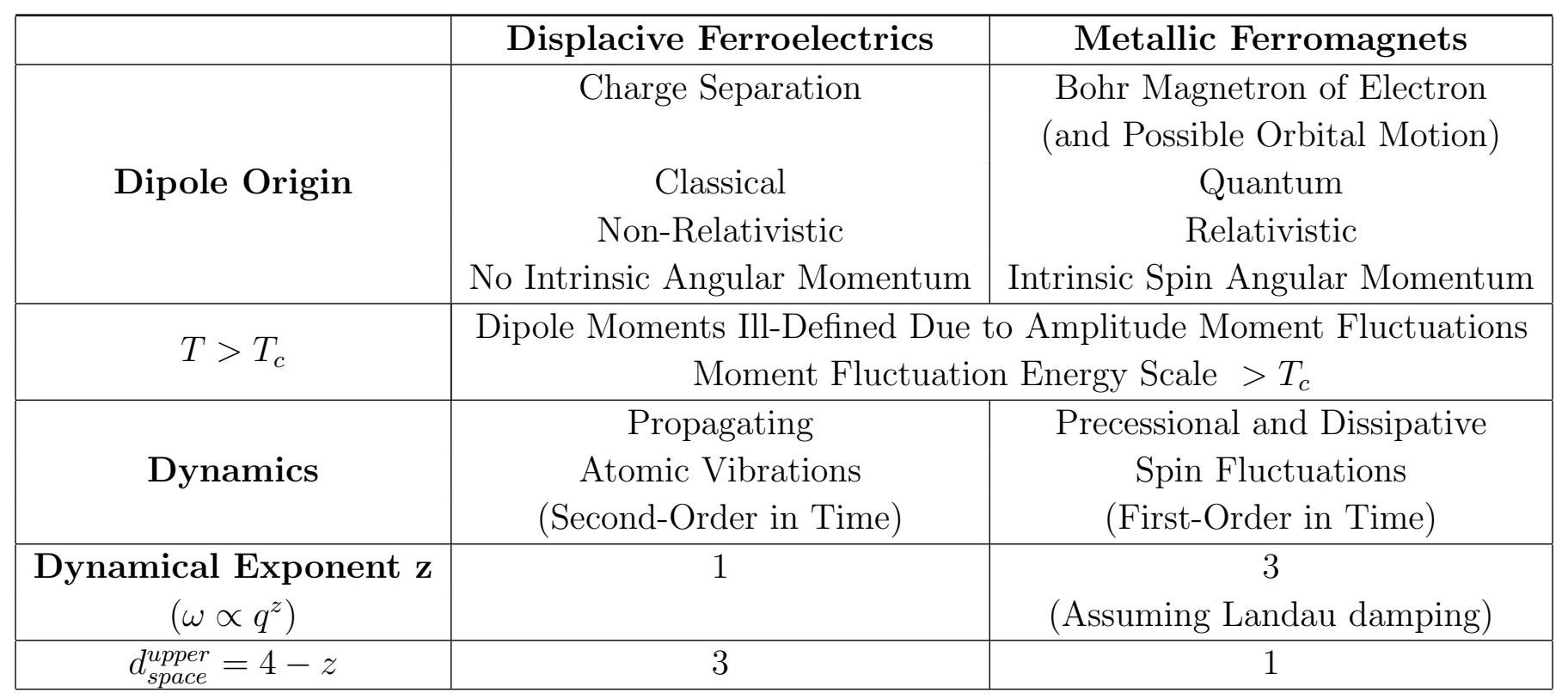

Table II: Key Similarities/Differences between Displacive Ferroelectrics and Metallic Ferromagnets Most Relevant for the Study of Quantum Criticality.

\section{The Case of $\mathrm{SrTiO}_{3}$ to Date}

So far we've discussed quantum criticality in displacive ferroelectrics in rather broad, abstract terms...let's now turn to what all this means specifically for the case of $\mathrm{SrTiO}_{3}$ (STO), a material that has been an important setting for basic research and for specific applications over the course of several decades [28, 73]. Here we will focus mainly on summarizing its low-temperature properties, where more detail can be found in reviews (and references therein) elsewhere [8, 9, 28, 73, 74]. As we have already discussed, ferroelectricity in the $\mathrm{ABO}_{3}$ perovskites is driven predominantly by soft longwavelength transverse optical (TO) phonons; thus this displacive ferroelectric (DFE) phase transition is naturally sensitive to pressure-tuning and hence to studies of quantum criticality. $\mathrm{BaTiO}_{3}$ (BTO) was the first perovskite ferroelectric to be identified, and the development of FE from its simple high-temperature cubic perovskite structure was very appealing and led to intense study [28]. At high temperatures, the dielectric response of $\mathrm{SrTiO}_{3}$, an isovalent cousin of BTO, is Curie-Weiss and suggests a ferroelectric temperature of $T_{c} \sim 40 K$. Like BTO, STO has a soft TO mode such that $\epsilon^{-1} \propto \omega^{2}$ over a broad temperature region [43]. However at $T_{c}=105 K$, STO has a cubic-tetragonal (C-T) transition where both phases are paraelectric in contrast to the $\mathrm{C}-\mathrm{T}$ transition 
in BTO where FE develops. In STO there are clear thermodynamic anomalies at $T_{c}$ but no inversion symmetry-breaking, though at low temperatures boundaries between tetragonal domains are polar $[75,76]$. Phonon softening at the Brillouin zone boundary is observed at $T_{c}$ and this antiferrodistortive (AFD) transition in STO is associated with the development of staggered rotations of oxygen octahedra in adjacent unit cells. Though STO polar soft modes are present, ferroelectricity is not observed to the lowest temperatures measured at ambient pressure [22].

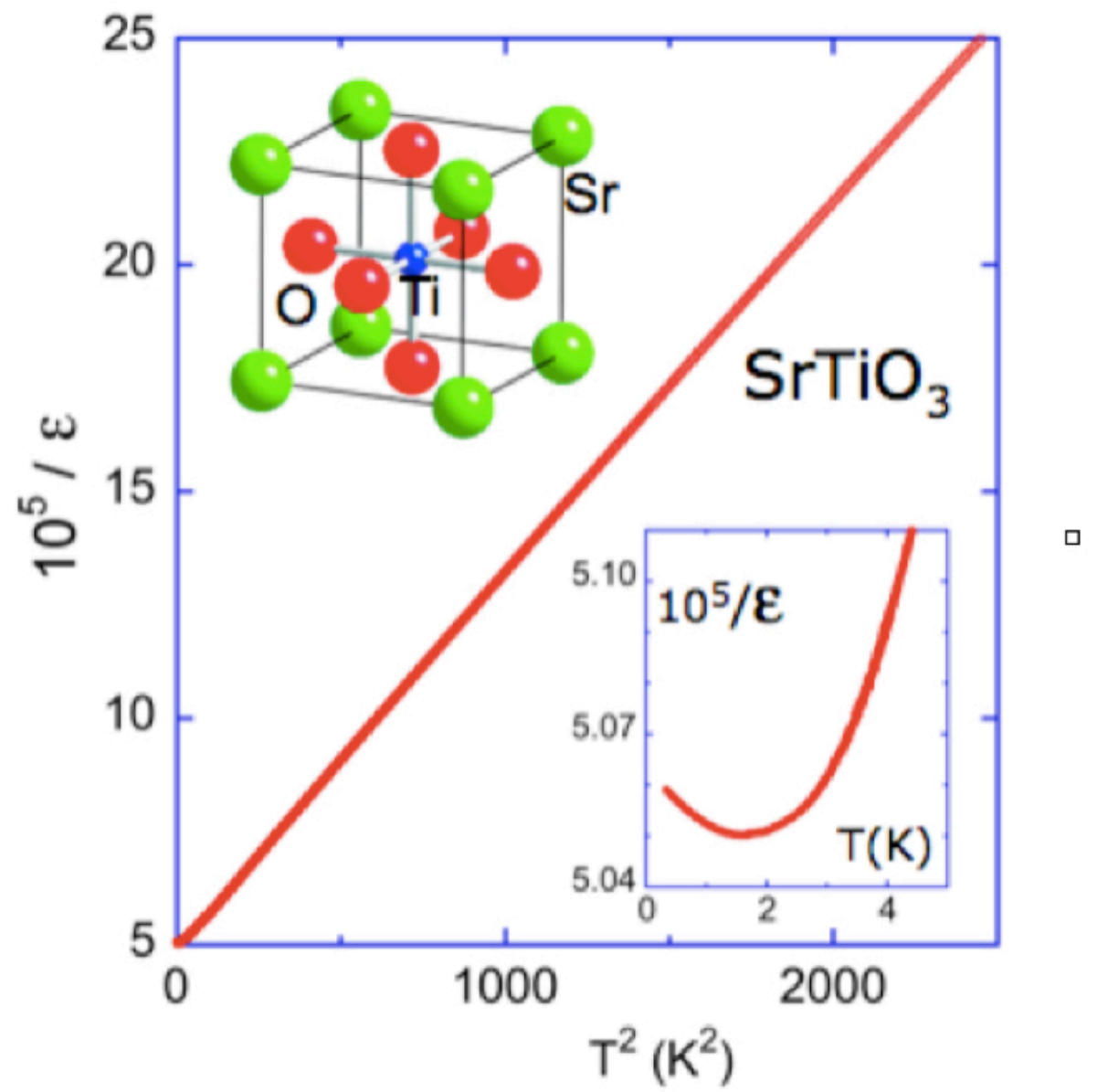

Figure 7. Temperature-dependance of the inverse dielectric function $\epsilon^{-1}(T)$ at ambient pressure for $\mathrm{SrTiO}_{3}$ as a function of the square of the temperature up to approximately $T=50 \mathrm{~K}$ from [22] indicating good agreement with the behavior $\epsilon^{-1} \propto T^{2}$ expected theoretically $(\epsilon=1+\chi)$ in the approach to a $d=3$ ferroelectric quantum critical point where the weak logarithmic corrections are not observed $[16,17,18,19,20,22,59,60]$. The room-temperature cubic perovskite crystal structure of $\mathrm{SrTiO}_{3}$ is shown in the top left corner. The lower inset is an expanded view of the low-temperature data [22], indicating an upturn below $4 K$ most likely due to coupling of the polarization with acoustic phonons $[19,22,59,77,78]$.

The unexpected low-temperature behavior in the dielectric response of STO (it 
is large but finite as shown in Figure 7) led to STO being named the first "quantum paraelectric" [79]. It was assumed that the stability of the paraelectric state in low temperature STO is due to effects of zero-point fluctuations analogous to the situation in liquid helium where crystallization is never achieved at ambient pressure. There was already prior theoretical literature on the effects of quantum fluctuations on low temperature displacive transitions $[16,17,59,60,62]$, and experiments on STO stimulated more theoretical research in this direction $[9,18,19,20,22,80,81,82]$. Usually one associates zero-point fluctuations with light atoms like hydrogen or helium so their significance for STO may seem surprising. However quantum effects can also assume importance when there are two or more low-temperature phases present, for example paraelectricity and ferroelectricity, with negligible energy differences [28]. In the case of STO, the coupling between the oxygen rotations and the soft polar mode is very small so that quantum fluctuations can affect the AFD and the FE effectively independently [78]; computationally quantum fluctuations have been shown to suppress the FE transition [81], supporting the proposal that STO is a quantum paraelectric. It was noted early on that the Einstein-Barrett expression (32) [62] for the dielectric susceptibility does not work well for STO [79], most likely because STO has a phonon dispersion [28]. Indeed it is exactly why STO is of interest to us at low temperatures since we expect scale-free quantum fluctuations there to be quite important.

The antiferrodistortive transition in STO at $T_{c}=105 \mathrm{~K}$ at ambient pressure is very close to a tricritical point and indeed STO is a marginal system very close to the stability edge of its paraelectric phase. External perturbations including uniaxial stress, epitaxial strain and chemical subsitution induce ferroelectricity at finite temperatures. More recently it has been found $[9,83,84,85]$ that ferroelectricity can also be induced in STO with isotope subsitution (Oxygen-18) such that for $\operatorname{SrTi}\left({ }^{16} \mathrm{O}_{1-x}^{18} \mathrm{O}_{x}\right)_{3}$ the ferroelectric transition temperature scales as $T_{F E} \propto\left(x-x_{c}\right)^{0.5}$ for $x \geq x_{c} \approx 0.3$ where $T_{F E}=23 K$ for $x=1$. In the simplest models isotope subsitution softens the polar phonons, and there are several such theoretical discussions specific to STO $[9,86,87,88]$; here the key assumption is that the mass increases at constant stiffness. However we might also expect that a decrease in frequency increases the susceptibility and thus decreases the stiffness, leading to an increase in fluctuation amplitude. The relative importance of mass vs. stiffness change in describing isotopic substitution in STO is a topic of current discussion.

On the experimental side, application of hydrostatic pressure to STO-18 $(x=1)$ suppresses its ferroelectric transition to zero-temperature [89], so that the effects of quantum fluctuations can be studied precisely at the QCP. More recently the dielectric response of $\operatorname{SrTi}\left({ }^{18} \mathrm{O}_{x}^{16} \mathrm{O}_{1-x}\right)_{3}$ has been studied for varying $x$ at very low temperatures at ambient pressure; because it does not depend strongly on sample growth conditions or purity, it has been suggested that disorder is not a key feature [22]. The detailed behavior of the dielectric response is in excellent agreement with theoretical predictions $[9,17,18,19,20,22,59,60,77,82]$, suggesting that this is a system where detailed interaction between theory and experiment are possible. Work is currently in progress 
on the Grüneisen ratio [66] in this same set of materials to explore its behavior at and in proximity to the DFE-QCP (displacive ferroelectric quantum critical point) [67]. We note it is necessary to take account of the coupling of the electronic polarization field with the acoustic phonons to obtain a full description of the dielectric behavior particularly at the very lowest temperatures, below a few Kelvin [19, 22, 77, 78].

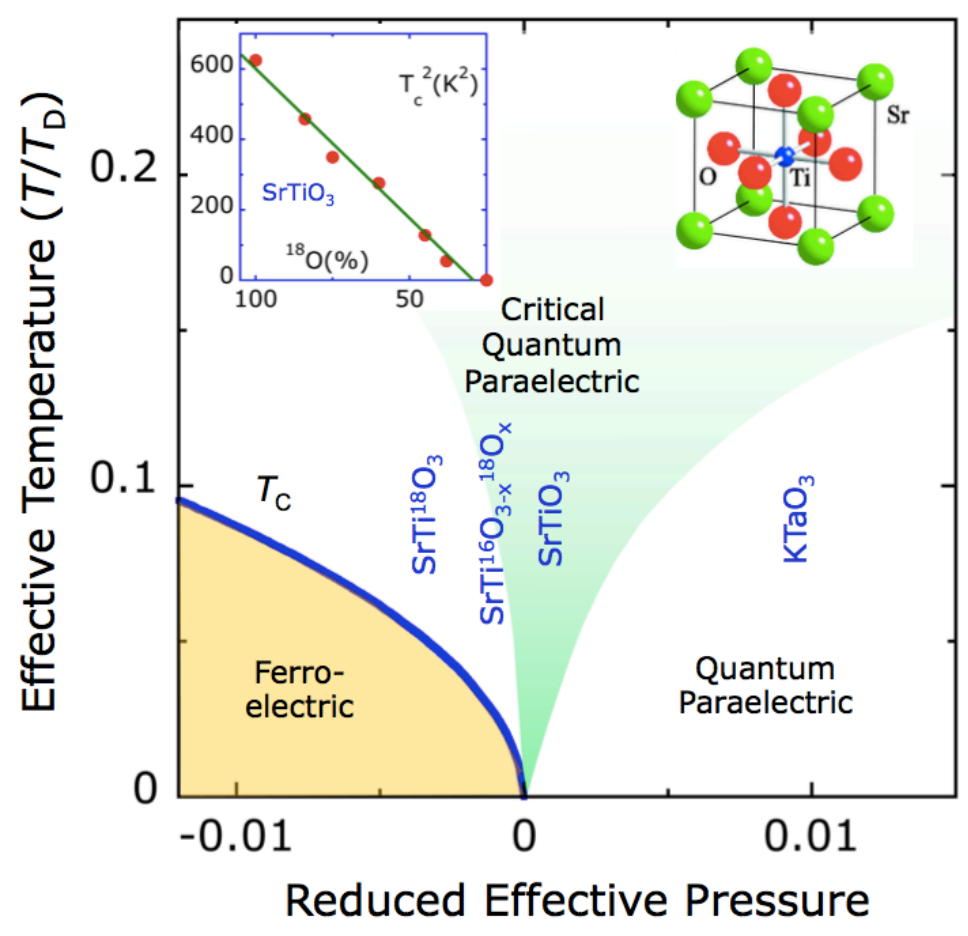

Figure 8. Effective temperature vs. reduced effective pressure phase diagram for $\mathrm{SrTiO}_{3}, \mathrm{KTaO}_{3}$ and related materials. Here the effective temperature is the ratio of the temperature and the material's Debye temperature associated with its optical phonon branch $\left(\frac{T}{T_{D}}\right)$. The effective pressure can be tuned by isotopic $\left(\operatorname{SrTi}\left({ }^{18} \mathrm{O}_{x}^{16} \mathrm{O}_{1-x}\right)_{3}\right)$ or by chemical $\left(\mathrm{Sr}_{1-x} \mathrm{Ca}_{x} \mathrm{TiO} \mathrm{O}_{3}\right)$ substitution [90, 91], or by application of external hydrostatic pressure [89]. Based on an integrated theoretical-experimental approach [22], a selection of materials is positioned on this phase diagram (with units of effective pressure defined in [22]) where a critical quantum paraelectric is one with a gapless dispersion $\left(\omega \propto q^{z}\right)$ whereas the Einstein-Barrett description [62] may only apply to materials in the "quantum paraelectric" phase with a gapped spectrum. Insert: $T_{c}^{2}$ vs ${ }^{18} \mathrm{O}$ percentage in $\operatorname{SrTi}\left({ }^{18} \mathrm{O}_{x}^{16} \mathrm{O}_{1-x}\right)_{3}$ with a linear slope indicating an isotopicallytuned ferroelectric phase transition temperature with $T_{c} \propto \sqrt{x}$, a result in agreement with self-consistent mean-field theory [22]. The room-temperature cubic perovskite structure of $\mathrm{SrTiO} 3$ is also shown in the top of the phase diagram. This figure is adapted from Rowley et al. [22].

For the sake of completeness, we should add that although the transverse optic soft mode in $\mathrm{SrTiO}_{3}$ reaches zero frequency only in STO-18 causing ferroelectricity 
below roughly $30 K$, there is a different and rather unexpected kind of short-range ferroelectric distortion in all isotopic variations of STO: below roughly $80 \mathrm{~K}$, the $\mathrm{Sr}$-ions displace along [111] directions, yielding a triclinic structures with local polarizations $[75,76]$. Under normal conditions, these local polarization cannot all be aligned to yield a macroscopic polarization, so in some important way cryogenic STO with ${ }^{18} \mathrm{O}$ does not behave as a conventional paraelectric. The ferroelectric nanodomains are nestled inside larger ferroelastic domains ("walls within walls") [75]. This local symmetry may play a role in the crystallographic structure of ferroelectric STO with ${ }^{18} \mathrm{O}$, and this remains an oBpen question. Again we note that the response time of these domains appears to be very slow [61] as they don't appear to contribute to observed low temperature thermodynamic quantities studied so far [22].

In a nutshell, STO and its isotope variants, provide a nice setting to study quantum criticality since the dynamics are simple (propagating) and it resides at its upper critical spatial dimension $d_{\text {space }}^{\text {upper }}=4-1=3$ so that results from both scaling and self-consistent phonon theories apply (up to logarithmic corrections) and can be compared in detail with experiment. In Figure 8 we display a schematic Temperature-Pressure phase diagram indicating the observed behavior of $\mathrm{SrTiO}_{3}$ and related perovskite materials at ambient pressure. Of course there are a number of other exciting recent developments associated with STO at low temperatures that also present exciting research opportunities both for fundamental study and also towards applications, and we mention them briefly here:

- Giant Piezoelectricity. The large piezoelectric response of STO at low temperatures makes it very useful for a number of cryogenic applications [92]. To our knowledge, the piezoelectricity of the isotopically mixed STO family has not been systematically measured and it may be tunable as a function of the ${ }^{18} \mathrm{O} /{ }^{16} \mathrm{O}$ ratios and epitaxial strain to suit specific needs.

- Photoinduced Enhanced Dielectric Constant. It has been found that a significantly enhanced dielectric constant can be induced in STO by ultraviolet radiation with the suggestion that it is related to quantum effects [93, 94], possibly through large polaron formation [95]

- Chemically Doped STO. There has been extensive work on the low temperature properties of chemically doped quantum paraelectrics [96], particularly on impurityinduced ferroelectricity. The development of quantum relaxors and quantum paraelectric glassiness has been less studied and could be important [97], as we'll discuss in the next section, for electrocaloric applications.

- Electron Transport in Doped STO. Electron transport in n-doped $\mathrm{SrTiO}_{3}$, achieved either by oxygen reduction or by Nb subsitution, has been observed [98], with high carrier mobility [99, 100] and unusual resistive behavior [101]. The magetoresistance and the Hall resistivity associated with photoinduced carriers in STO is also unconventional [102] suggesting that the metallic state emerging from doped STO may need further characterization particularly due to its very low Fermi temperature. 
- Superconductivity in STO. Electron-doped STO is one of the most dilute superconductors known [103, 104], and most likely a non-BCS mechanism is necessary for its explanation More recently a gate-tunable insulatingsuperconducting transition has been observed in an STO weak link [105], again pointing to anomalous behavior in this material. The dependence of the superconducting $T_{c}$ on the percentage of ${ }^{18} 0$ in the STO is an active topic of theoretical [106, 107] and experimental [108] research. We will return to the question of superconductivity in STO in the "Open Questions" section.

These are just some of the many stimulating questions associated with STO at low temperatures. Of course this material is very much in the news at higher temperateratures including its role in oxide interfaces $[109,110,111]$ and as a substrate that mysteriously enhances the superconductivity in FeSe [112].

In this section we have focussed on ferroelectric quantum criticality in STO, and we conclude it by noting that ferroelectric quantum phase transitions have been observed in a variety of systems including other insulating perovskites [113], organic complexes $[114,115,116]$ and narrow-band semiconductors [117]. In order to emphasize this point, in Figure 9 we display four distinct examples of ferroelectric quantum transitions, noting the range of $T_{c}$ 's accessible with chemical substitution and applied pressure.

\section{A Flavor for Low Temperature Applications}

Let us now turn to some low-temperature applications of ferroelectrics. As we mentioned earlier, the current trends due to market demands are for faster and smaller devices. Ferroelectric films are used as passive elements in dynamical random access memories (DRAMs) comprised of grids of capacitors with access transistors; here each bit is stored in a distinct capacitor where 0 and 1 correspond to the absence/presence of charge $[36,118]$ and the appeal of FE (and PE) materials is their high dielectric constants. DRAMS are among the highest density memories in current use with readily available 64 Gbit chips. Despite their many attractive features that include ultrafast speeds and low cost, DMRAMs require regular memory refresh cycles to ensure that the stored data is not lost due to everpresent leakage currents. The refresh interval, currently about 60 milliseconds, depends on the ratio of the stored charge to the leakage current. An area of current interest is to lengthen the time between refresh cycles, both to increase device time for memory access and to reduce power consumption. If such a "long-refresh DRAM" were run at $77 \mathrm{~K}$, where the leakage currents are significantly smaller than at ambient temperature, the refresh frequency might drop orders of magnitude from $\mathrm{kHz}$ to $\mathrm{Hz}$ where details would depend on material specifics.

Ferroelectric films are also used as active memory elements in FeRAMs (ferroelectric random access memories, also called FRAMS) where information is stored in polarization (charge) states $[28,36,119]$. The low cost and high speed of FeRAMs makes them competitive with other storage devices [36, 119] if they can maintain the demands of miniaturization [120]; they are particularly attractive for satellite applications due 
a)

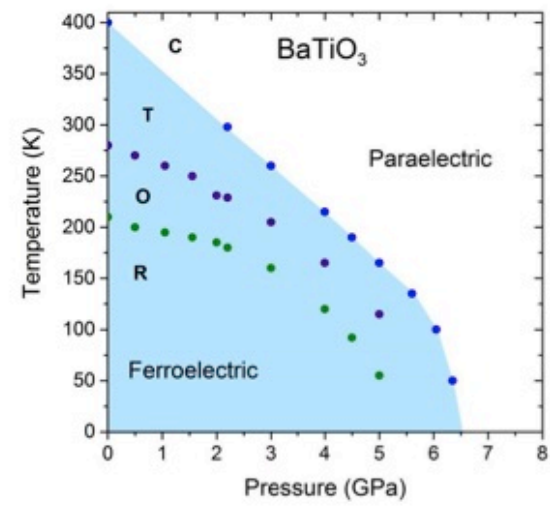

c)

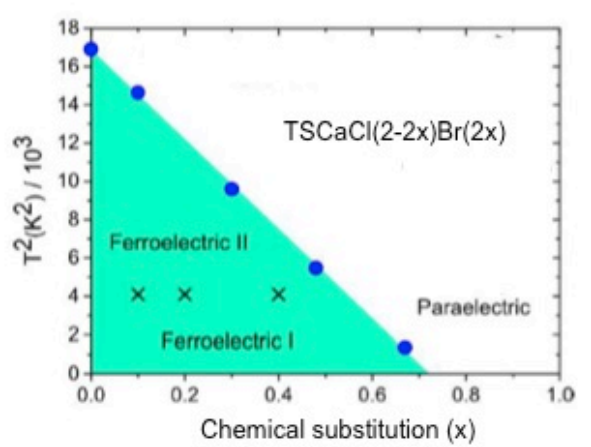

b)
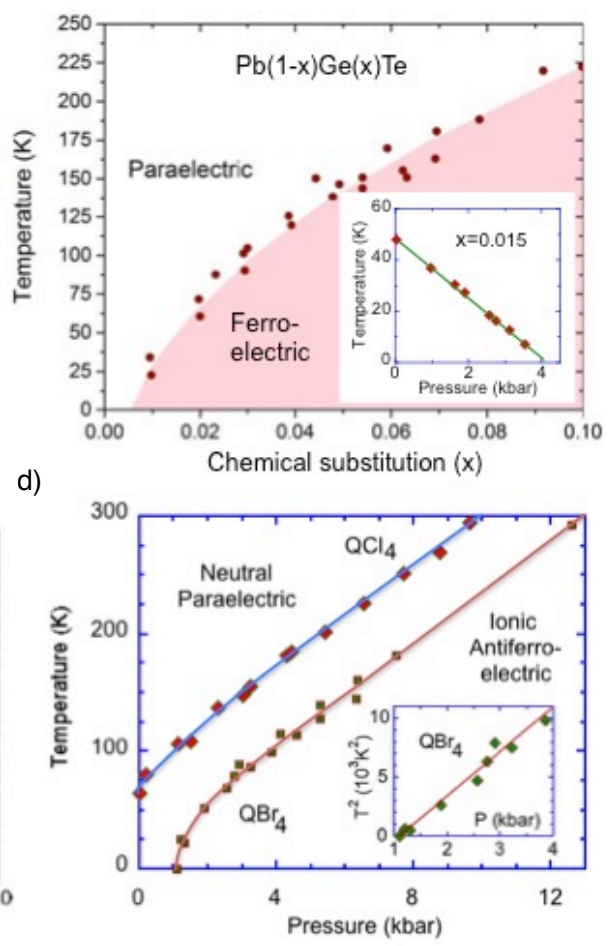

Figure 9. Four phase diagrams indicating different materials where ferroelectric quantum phase transitions have been studied experimentally with tuning by pressure or by chemical substitution. a) Pressure-tuned ferroelectric quantum phase transition in perovskite $\mathrm{BaTiO}_{3}$. The figure labels $\mathrm{C}, \mathrm{T}, \mathrm{O}$ and $\mathrm{R}$ refer to the cubic, tetragonal, orthorhombic and rhombohedral structural phases of $\mathrm{BaTiO}_{3}$. The polarization direction points in different directions in each of the three ferroelectric phases ( $\mathrm{T}, \mathrm{O}$ and $\mathrm{R}$ ). All transitions are firstorder at ambient pressure. This figure is adapted from Ishidate et al. [113] with permission. b) The IV-VI family of narrow-band semiconductors GeTe and PbTe have soft transverse-optical phonon modes that can lead to ferroelectric instabilities. Pressure, carrier concentration and chemical composition can be used to tune these materials through ferroelectric quantum transitions as shown in this figure adapted from Suski et al. [117].c) Quantum phase transition in a compositionally tuned organic uniaxial ferroelectric tris-sarcosine calcium chloride. Here the quantum ferroelectric transition is tuned by chemical substitution. This figure is adapted from Rowley et al. [116]. d) Pressuretemperature phase diagrams of the charge-transfer complexes $D M T T F-Q C l_{4}$ and $D M T T F-Q B r_{4}$. Inset: Close to $P_{c}, T_{c}^{2}$ scales with $P$ in the ionic antiferroelectric $D M T T F-Q B r_{4}$. We note that this scaling is similar to that of $T_{c}(x)$ shown in the inset of Figure 9 , suggesting that external and chemical pressure have similar effects on $T_{c}$. This figure is adapted from Horiuchi et al. [114] with permission. 
to their radiation hardness [36]. Data storage cells in FeRAMs, as in DRAMs, consist of ferroelectric capacitor-based structures with access transistors; in FeRAMs it is the nonlinear relationship between applied field and polarization (charge) in ferroelectric materials that is exploited to store information analogous to the situation in magnetic core memories. For such a memory cell, the switching barrier $(\Delta U)$ must be larger than the thermal energy scale, $k_{B} T$, so that the stored information is not corrupted. Therefore we can equate the switching and the thermal barriers

$$
\Delta U=k_{B} T \quad \Rightarrow \quad L_{c}
$$

to obtain a critical length-scale $L_{c}$ that sets the lower-bound on the characteristic system size. In a ferroelectric memory, the switching barrier can be estimated as the energy stored in its effective capacitor. Since these devices are operated at fixed voltage $(V$, the standard silicon logical level that is currently $4.5 \pm 0.5$ volts) with effective capacitance $C$, we write

$$
\Delta U=\frac{1}{2} C V^{2} \propto L \quad(\text { for fixed } \quad V)
$$

so that we see that the switching barrier scales with $C$ and hence with its characteristic length [36]. More specifically, taking $C=\left(\epsilon_{0} \epsilon\right)(\alpha)$ where $\alpha=\frac{A}{d}$, we find that

$$
L_{c}=\left(\frac{T}{V^{2}}\right)\left(\frac{4}{\alpha \epsilon}\right) 10^{-13} \mathrm{~m}
$$

where $T(K), V(v), \alpha(m)$ and $\epsilon$ are inputs. A typical FRAM currently available uses PZT (lead zirconate titanate, $\mathrm{Pb}(\mathrm{Zr}, \mathrm{Ti}) \mathrm{O}_{3}$, with $\epsilon=1300$ ) and operates at ambient temperature $(T=300 \mathrm{~K})$ with $\alpha=10^{-5} \mathrm{~m}$ since it is $100 \mathrm{~nm}(L)$ thick with a lateral length of about 1 micron; at the current voltage standard ( 5 volts), $L_{c}$ is $0.1 \mathrm{~nm}$ $\left(L \gg L_{c}\right)$ indicating that these FRAMs are thermally safe. However should $V, \alpha$ and/or $\epsilon$ decrease in the future, $T$ is a very useful tuning parameter that can be reduced to ensure that the stored charge is robust to thermal fluctuations. In Figure 10 we show the scaling of the characteristic length $L_{c}$ for three specific materials at room temperatures using current device parameters.

Reduced operating temperatures leads to decreased conductivities and thus to increased breakdown fields [36]. Higher $E$ fields can then be applied, resulting in increased charge and hence enhanced signal to noise for the sense amplifiers [36]; we recall that the relative polarization is the switched charge per unit area. Typically this is determined by applying a series of voltage pulses before and after the switching. The resulting currents are measured over time and and these integrated curves determine the switched charge [36, 37]. Because the voltage is fixed at a standard logic level, increased electric fields require decreasing the FE film thicknesses. However if we try to increase stored charge by making a FE film thinner at room temperature, it may short since its conductivity is too high to prevent breakdown. More generally, the breakdown threshhold depends on the product of the electric field and the conductivity $(\sigma)$ or rather on the ratio $\frac{V \sigma}{d}$ [36]. Therefore for fixed $V$, we can reduce the film thickness $d$ if we also decrease $\sigma$ which is achieved by lowering the ambient temperature. 


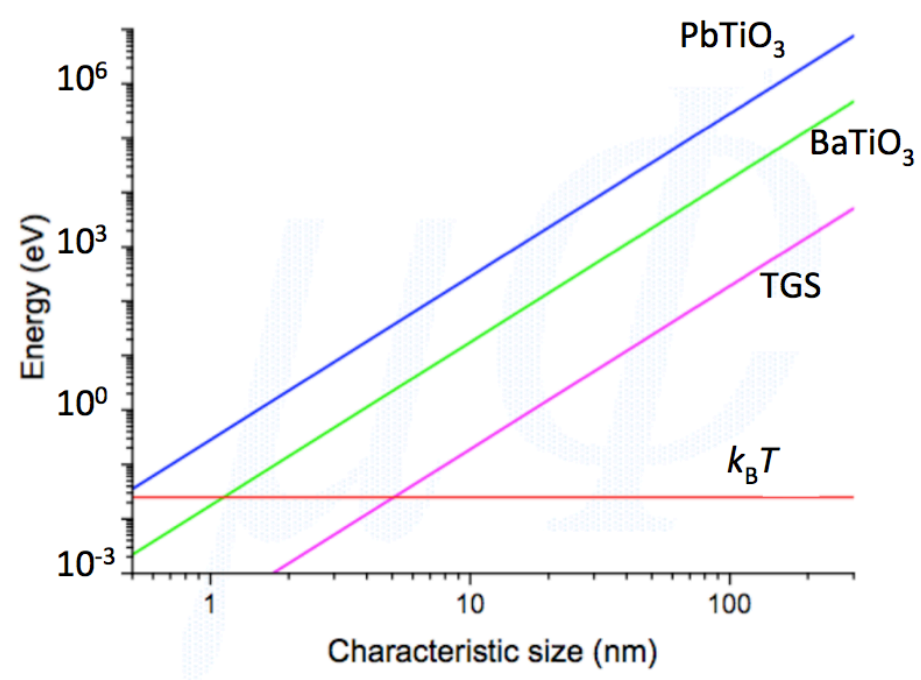

Figure 10. The minimum device size for room-temperature operation without thermal corruption for three different materials where TGS stands for triglycine sulfate; reproduced from M. Alexe [121] with permission and thanks.

Luckily ferroelectrics themselves can play a role in refrigeration via electrocaloric cooling (EC), the reduction of temperature of a FE material in response to the removal of an electric field $[28,33,36,122,123,124]$. Its magnetic counterpart, magnetocaloric cooling (MC), if often used to access millikelvin temperatures. Until recently EC effects were too small for practical applications and thus were not pursued. However several developments $[122,123,124,125]$, suggest that we should revisit this phenomenon, particularly at low temperatures. More specifically the breakdown fields of FE films are significantly larger than those of their bulk counterparts so that higher $E$ fields can be applied, and multicapacitor technology can be used to increase their effective volumes [123]. But we are getting ahead of ourselves. In the spirit of being self-contained, let's remind ourselves of the key features of adiabatic cooling so that we can understand why to date the magnetic version has been more successful than its electric counterpart at low temperatures (and why we believe this topic deserves to be revisited!).

The entropy as a function of field and temperature $(S(E, T))$ plays a key role in the electrocaloric effect and its magnetic analogue (MC) where $E$ is replaced by $B$. We can write

$$
d S=\left(\frac{\partial S}{\partial T}\right)_{E} d T+\left(\frac{\partial S}{\partial E}\right)_{T} d E
$$

where, for an adiabatic process $(d S=0)$ and using the Maxwell Relation $\left(\frac{\partial S}{\partial E}\right)_{T}=\left(\frac{\partial P}{\partial T}\right)_{E}$ (where $P$ is the polarization), we obtain

$$
-\left(\frac{\partial T}{\partial E}\right)_{S}=\frac{\left(\frac{\partial P}{\partial T}\right)_{E}}{\left(\frac{\partial S}{\partial T}\right)_{E}}=T \frac{\left(\frac{\partial P}{\partial T}\right)_{E}}{c_{E}} .
$$

Here $c_{E}$ is the specific heat at fixed electric field that has contributions from polar $\left(c_{E}^{P}\right)$ and nonpolar $\left(c_{E}^{X}\right)$ modes, where the latter are predominantly acoustic phonons. The 
a)

b)

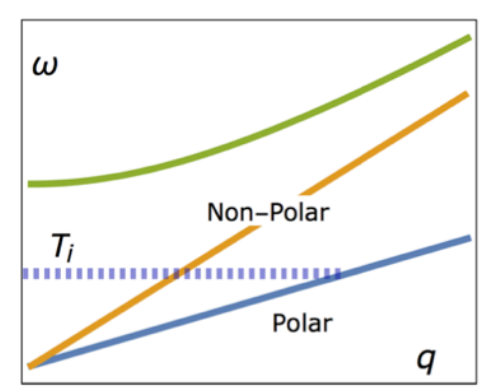

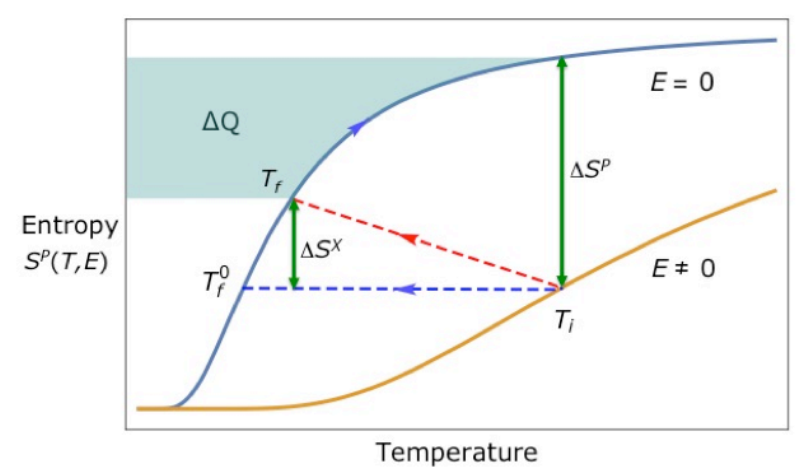

c)

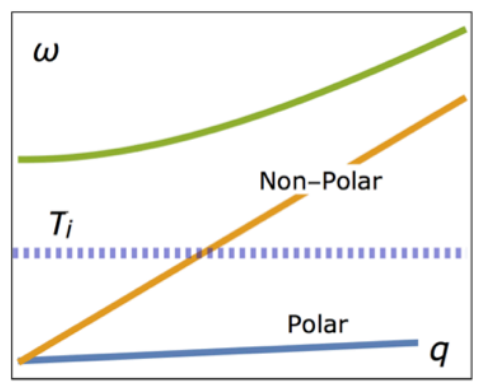

Figure 11. (a) $S^{P}(T, E)$ Entropy-temperature cycling for two distinct field strengths indicating the Carnot-like heat cycle that is the basis for electrocaloric cooling. Here $T_{i}$ is the initial temperature in the adiabatic depolarization process, $T_{f}^{0}$ is the final temperature in the absence of coupling to the nonpolar modes and $T_{f}$ is the final temperature including the effect of the nonpolar modes. $\Delta Q$ is the heat that can be extracted from an external load. We require $\Delta S^{P}$ to be substantially greater than $\Delta S^{X}$ for effective cooling to occur (as in case (c) in contrast to case (b). (b) Hypothetical dispersion where the sound velocity in the polar branch is less than that in the nonpolar branch. (c) Hypothetical dispersion where the polar modes have a relatively flat dispersion, indicating very low interactions between the ions or the dipoles and thus large polar entropy.

specific heat is of course a measure of the entropy and thus its magnitude will be related to the dispersion which together with the Bose function determines the distribution of low-energy excitations as a function of wavevector in the Brillouin zone. In a displacive $\mathrm{FE}$, the low-frequency polar modes are localized in q-space and $c_{E}^{P}$ is exponentially suppressed with a gap $(E \neq 0)$; thus at low temperatures $c_{E}$ is dominated by $c_{E}^{X}$ and varies as $T^{3}$. These same acoustic phonons, in the absence of a ferroelectric phase transition, are the main contribution to the pyroelectric coefficient $\left(\frac{\partial P}{\partial T}\right)_{E}$; it is expected to decrease sufficiently rapidly with decreasing temperature that $\left(\frac{\partial T}{\partial E}\right)_{S}$ in (44) vanishes in the limit $T \rightarrow 0$ [28]. Consistent with this argument, cryogenic studies of $\mathrm{KTaO}_{3}$ and $\mathrm{SrTiO}_{3}$ yielded negligible EC effects $[126,127,128,129]$ and this approach to low temperature refrigeration has not been actively pursued for some time. So why then can magnetocaloric cooling be used routinely to access very low temperatures in complete 
contrast to its electric counterpart (to date)?

We can address this question by looking at the entropy of the polar modes, $S^{P}(E, T)$, as a function of field and temperature shown schematically in Figure 11 a). Here we start at an initial temperature $T_{i}$ at $E=0$ and isothermally apply a finite electric field; the entropy of the polar modes is then lowered. The electric field is then removed adiabatically, and the temperature $T_{f}^{P}$ associated with the polar modes (uncoupled to other degrees of freedom) decreases. However since the total system is in equilibrium all modes, polar and nonpolar, must be at the same temperature. More specifically the total entropy $(S)$ is a sum of the polar and the nonpolar contributions, $S=S^{P}+S^{X}$ and there will be overall cooling of the system if and only the entropy $\Delta S^{P}$ is substantially greater than $\Delta S^{X}$ in Figure 11 a: more to the point, a nonzero $\Delta S^{P}$ is not good enough! In other words, the polar entropy loss due to the applied electric field must exceed the entropy to be removed from the acoustic phonons; in this case the system is cooled to a final temperature $T_{f}$ such that $T_{f}^{P}<T_{f}<T_{i}$ as shown schematically in Figure 11 a). This is difficult to achieve in simple displacive ferroelectrics where the sound velocity of the polar modes is not substantially below that of the nonpolar ones. One way to obtain $\Delta S^{P}>>\Delta S^{X}$ might be to reduce the sound velocity in the polar branch significantly compared to its nonpolar counterpart (see Fig. $11 \mathrm{~b}$ ), effectively reducing the coupling between electric dipoles to increase their entropy. Another approach to $\Delta S^{P}>\Delta S^{X}$ is to identify materials where the polar modes have flat dispersion bands, again indicating low dipole-dipole effective interactions and a high polar entropy. (see Fig. $11 \mathrm{c}$ ). We note that such flat dispersions are signature features of spin systems, specifically dilute paramagnetic salts and frustrated magnets, that are commonly used in cryogenic solid-state refrigeration [130]. Because the dipoledipole interaction is typically several orders of magnitude larger for electric dipoles than for their magnetic counterparts [29], the identification of paraelectric and ferroelectric materials with the necessary high polar entropy at low temperatures is particularly challenging.

What about electrocaloric cooling at low temperatures near a ferroelectric quantum critical point? Interestingly enough, this question has already been posed near a magnetic quantum critical point [131], and work is currently in progress to study the FE case [132]. Ideally we want a system with a high density of minimally coupled electric dipoles at low $T$ to achieve an enhanced polar entropy; possible candidates include order-disorder, relaxor materials and ferrielectric materials. Ideally we'd be approaching a quantum tricritical point to maximize the change in polarization without hysteresis; if we want the sound velocity of the polar modes to approach zero, then we also want to be at a Lifshitz point. Furthermore we'd like the system to have a uniaxial polarization to maximize coupling to the electric field $(\vec{E} \cdot \vec{P})$. Amnonia sulphate is an order-disorder ferroelectric with a high entropy at its FE transition, though it has not been practical for EC at room temperature due to its ionic conductivity [123]. This may not be an issue at low temperatures where ionic motion becomes frozen [123]. Indeed, analogous to their magnetic counterparts, dilute paraelectric salts have been used to cool small 
samples to millikelvin temperatures [133, 134, 135, 136]; with current multicapacitor technology this technique could be greatly improved and should be revisited.

In principle low-temperature electrocaloric cooling has many advantages over its magnetic counterpart, particularly its reduced size (no magnets necessary!) and its comparative simplicity of operation....we just have to find the right materials to make it work! Joule heating should not be a problem since the polar materials are reasonable insulators. For space applications, where dilution refrigeration is difficult to use particularly in microgravity conditions [131, 137], electrocaloric cooling has an additional advantage as FE materials are robust to everpresent cosmic rays [36].

Other possible applications for low-temperature paraelectric/ferroelectric materials include:

- Satellite Electronics. The radiation effects, due to cosmic rays and to solar activity, are not evenly distributed for low-Earth orbits and are even harsher in outer space. There is an urgent need for new electronics that are high-performance, radiation-tolerant and reliable [138] at an ambient temperature of roughly $10 K$, and onboard infrared detectors require $m K$ operating temperatures.

- Phased-Array Radar. Ferroelectric-superconductor "sandwiches" hold promise as phase shifters in phased-array radar $\mathrm{GHz}$ devices, running at significantly lower voltages than current versions. The dielectric losses must be kept very low to be competitive with existing bulky technologies and thus they would have to be run at low temperatures [36], possibly maintained by electrocaloric cooling.

- High Permittivity Supercapacitors. There is an increasing need for high density storage of electrochemical energy with rapid charge/discharge cycles and long lifetimes. Low dielectric loss and large-scale requirements could make this a niche for low-temperature PE/FE materials that are relatively cost effective [139]

and there are certainly many more!

\section{Open Questions for Future Research}

In order to emphasize research prospects, we conclude with a list of open research questions in this area of materials near ferroelectric quantum phase transitions:

\section{- Specific FE Materials for Study at Low Temperatures.}

Here we have argued that the study of materials near their ferroelectric quantum critical points (FE-QCPs) can play an important role towards understanding universality at quantum phase transitions. However there are only a few systems currently known that remain paraelectric to the lowest temperatures, so are those the only materials in this class to study? There are certainly many materials with low (classical) ferroelectric transition temperatures $\left(T_{c}<100 K\right)[28,36]$, and we expect that these $T_{c}$ 's could be reduced with pressure, stress or with 
chemical or isotopic substitution to yield possible QCPs that, to our knowledge, have not yet been explored. Empirically it seems that ferroelectric transition temperatures are very sensitive to pressure, as shown in Figure (9 a) with the case of $\mathrm{BaTiO}_{3}$. If this pressure-sensitivity of $T_{c}$ is indeed the general case, then this would significantly broaden the range of materials $[28,36]$ where quantum phase transitions could be studied. Furthermore the possibility of antiferroelectric quantum criticality could be pursued in materials like $\mathrm{NaNbO}_{3}$ with coexisting ferroelectric and antiferroelectric interactions [140] whose low antiferroelectric $T_{c}$ $(\sim 12 K)$ could be reduced (e.g. by substitution) and where quantum fluctuations are known to be important at low temperatures [141]. We note that competing energy- and length-scales can lead to quantum electric-dipole liquids [142], novel textures $[143,144,145,146]$ and exotic topological excitations $[77,147]$ in the vicinity of these quantum phase transitions.

\section{- Add Spin: A Multiferroic QCP.}

Additional degrees of freedom can be added in a systematic fashion to materials near their ferroelectric quantum phase transitions with rich phase behavior expected [148]. For example, quantum criticality in multiferroic materials [149] is only starting to be explored [30, 150, 151, 152, 153, 154] where the possible interaction of two quantum critical points could lead to novel behavior. Of course here we have been predominantly discussing bulk materials, but the low temperature behavior of multiferroic heterostructures [155, 156] could be intriguing as well. Multiferroics at low temperatures with high polar and spin entropies could also be candidates for advanced cryogenic solid-state refrigeration [157] based on both the electrocaloric and the magnetocaloric effects. We also note the intriguing case of multiferroic relaxor quantum critical points $[116,154]$, that may be related to quantum glassiness.

\section{- Add Charge: An Exotic Metal and Unusual Superconductivity}

The study of quantum criticality in magnetic metals is often motivated by the search for non-Fermi liquids and for unconventional superconductivity [14]. It is thus fitting that we note that the study of materials near a FE-QCP also fits into this "grand scheme."

Charge is another degree of freedom that can be added to a material near its FE-QCP by either chemical and/or gate doping. The Mott criterion [158] for the critical dopant concentration $\left(n_{c}\right)$ for a metal-insulator transition in doped semiconductors occurs when the average dopant-dopant distance $\left(d=n^{-\frac{1}{3}}\right)$ is a significant fraction of the effective Bohr radius $\left(a_{B}^{*}=\frac{\epsilon \hbar^{2}}{m^{*} e^{2}}\right)$ where $\epsilon$ is the dielectric constant; more concretely the critical concentration $n_{c}$ is defined as $n_{c}^{\frac{1}{3}} a_{B}^{*} \approx 0.26$, 
consistent with experiment in many semiconductors [159]. Since the effective Bohr radius is proportional to the dielectric constant $(\epsilon)$, it is much larger in n-doped STO than in doped semiconductors based on silicon or germanium (see Figure 12); therefore a lower $n_{c}$ is expected, consistent with observation [103, 159].

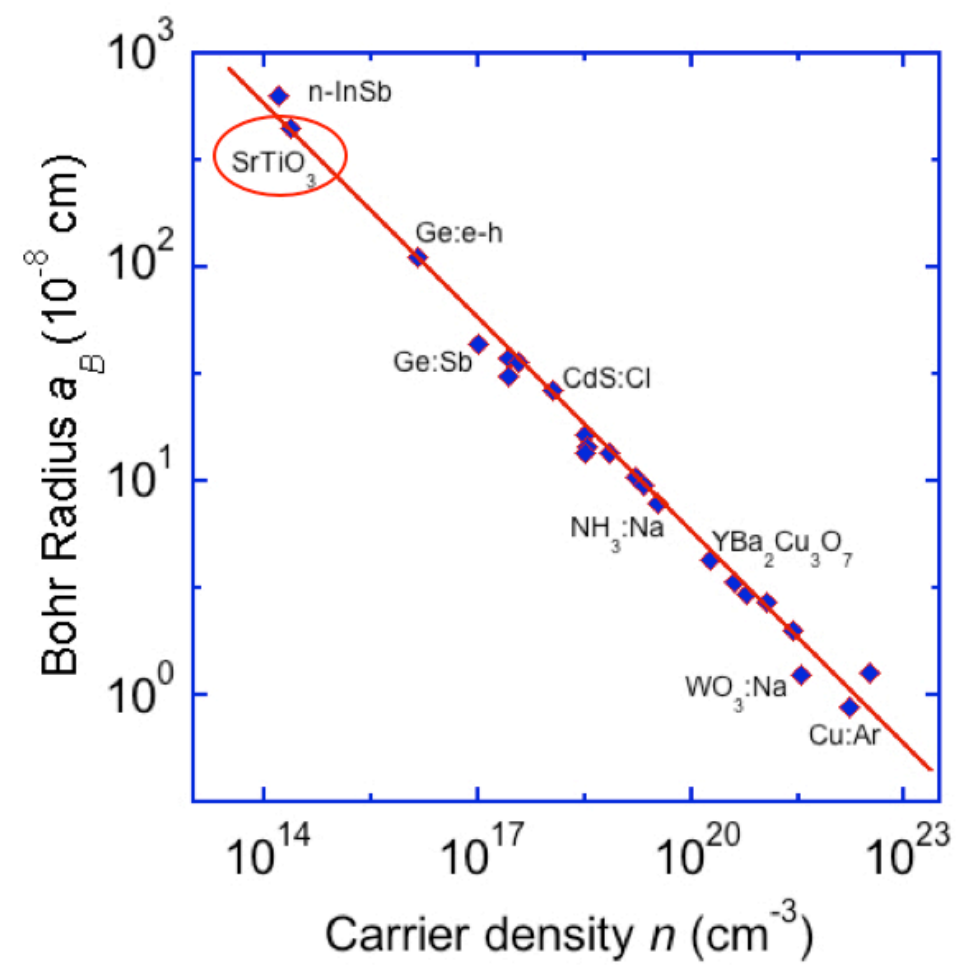

Figure 12. A plot of the effective Bohr radius $\left(a_{B}\right)$ vs. carrier density $(n)$ indicating good comparison between the Mott criterion $\left(n_{c}^{\frac{1}{3}} a_{B}=0.26\right)$ for the metal-insulator transition and experimental systems where $a_{B}$ and the critical carrier density $\left(n_{c}\right)$ for metallicity are known. Because the effective Bohr radius is inversely proportional to the dielectric constant, it is large for $\mathrm{SrTiO}_{3}$ indicating a low critical carrier concentration for the metal-insulator transition consistent with observation [103]. This figure is adapted from Edwards and Sienko [159] with permission and with thanks to K. Behnia [160].

The Fermi temperature of metallic n-doped STO can be quite low because of the relatively high carrier effective mass and low densities of practical interest; for example for $n=5.5 \times 10^{17} \mathrm{~cm}^{-3}, T_{F} \approx 13 K$ [103]. At first sight this dilutecarrier metal looks quite conventional with a resistivity that scales like $T^{2}$ as expected for a three-dimensional Fermi liquid [161]. The catch is that this behavior continues to temperatures well above the Fermi temperature $T_{F}[101,161]$ where $T_{F}$ is determined from the coefficient of the linear heat capacity; in $3 \mathrm{~d}$ for fixed $m$, $T_{F}$ scales with $n^{\frac{2}{3}}$. Arguments based on Fermi liquid, requiring that $T<<T_{F}$, are clearly inapplicable for $T>T_{F}$; furthermore $A$, the coefficient of this $T^{2}$ behavior 
in the resistivity, can change by four orders of magnitude by tuning the carrier concentration and persists to dilute limits where known mechanisms for $T^{2}$ behavior are no longer applicable [101].

The traditional BCS theory of superconductivity [162] requires $T_{F} \gg T_{D}$ where $T_{F}$ and $T_{D}$ are the Fermi and the Debye temperatures, a condition not satisfied in n-doped STO; for $n=5.5 \times 10^{17} \mathrm{~cm}^{-3}, T_{F} \approx 13 \mathrm{~K}$ and $T_{D} \sim 400 \mathrm{~K}$ so that $T_{F} \ll T_{D}$ [103]. The possibility of superconductivity in doped paraelectric materials was considered within the decade after the BCS theory was developed [163], and it was originally suggested that in polar semiconductors the temperature-scale associated with the longitudinal optical phonon, $T_{L}$, could replace $T_{D}$ in the BCS formalism. However, because typically $T_{F} \ll T_{L}$ for densities $n \lesssim 10^{19} \mathrm{~cm}^{-3}$, the implication was that superconductivity in doped paraelectrics was unlikely [163]. Nevertheless superconductivity was predicted $[164,165]$ in n-doped STO based on intervalley scattering; this theory led to the experimental search and subsequent observation of superconductivity [166] in this material. Ironically, despite this finding, it was later shown that key aspects of the motivating theory, particularly the assumption of multiple valleys, were inapplicable to STO [167]; this unusual twist in the discovery of superconductivity in doped STO only makes its existence all the more remarkable $[106,107,168,169,170,171]$.

In summary superconductivity occurs in n-doped STO, and we still have a lot to learn about its underlying mechanism and the symmetry of its order parameter. It has been observed both in bulk [91, 103, 108, 172, 173, 174, 175] and, more recently, at the interface of $\mathrm{LaAlAs}_{\mathrm{SrTiO}}$ [176, 177, 178]. Like many of the heavy fermion superconductors, it is in the parameter regime $T_{F}<<T_{D}$ and thus cannot be described by conventional BCS theory; however here spin-fluctuation mediated pairing cannot be applied. Instead it is natural to consider electronelectron interactions mediated by long-range Coulomb potentials. However here there is a conundrum: the pairing interaction $V(\omega)$ scales inversely proportional to the dielectric constant $\epsilon(\omega)$ so that at $\omega=0$ the interaction is small (since $\epsilon(0)$ is large). We recall that, within a soft mode picture described by (20) and (21), the dielectric constant can be written as

$$
\frac{\epsilon(\omega)}{\epsilon_{\infty}}=1-\left(\omega_{L O}^{2}-\omega_{T O}^{2}\right) \frac{\omega_{T O}^{2}}{\left(\omega^{2}-\omega_{T O}^{2}\right)}
$$

where the transverse and longitudinal frequencies, $\omega_{T O}$ and $\omega_{L O}$, are defined by the zero and the pole of $\epsilon(\omega)$. We see that in the frequency window

$$
\omega_{T O}<\omega<\omega_{L O}
$$

$\epsilon(\omega)$ is negative leading to an attractive interaction $V(\omega)$; furthermore we note that this "attractive frequency range" is increased to its maximal value close to a FE-QCP where $\omega_{T} \rightarrow 0$. Here, for simplicity, we have suppressed the $q$-dependence of $V(\omega)$ and $\epsilon(\omega)$, but it is likely to be important due to the long-range nature of 
the Coulomb interaction. Furthermore we need to consider screening effects of the added carriers that become progressively more important with increasing $n$.

So here we have a dynamical interaction between the electrons... what's so difficult about this superconducting problem? Actually there are two challenges to address. The first is that a key aspect of Cooper's crucial superconducting pairing argument relies on being close to the Fermi energy [162]; in this case the pairing problem becomes effectively $2 \mathrm{~d}$ where, in contrast to $3 \mathrm{~d}$, binding is possible with an arbitrarily weak attraction. This reasoning is not applicable to n-doped STO where the pairing energy-scale is much higher than $T_{F}$. Second, any attractive pairing of electrons must somehow "bypass" their repulsive Coulomb interaction. In the BCS theory retardation is crucial [162]: the ionic screening cloud lags behind the electron, thereby mediating its attraction to other electrons. By contrast in n-doped STO, where there is no similar large separation of time-scales, further study of possible "Coulomb circumvention" mechanisms is needed. In a nutshell in superconducting n-doped STO we are without two key features of the successful BCS theory of superconductivity...how would the theoretical description of superconductivity have developed if this amazing phenomenon had first been observed in n-doped STO rather than in mercury?

Finally we should note that electron-doped STO is one of the most dilute superconductor known to date [91, 103]; its density of charge carriers, coming from niobium doping (on Ti sites), lanthanum substitution (on $\mathrm{Sr}$ sites) or from oxygen vacancies, is comparable to that of the metal bismuth that has only very recently been shown to go superconducting, albeit at a temperature much lower than that observed in n-doped STO [104].

Since much study of quantum criticality is motivated by the search for novel forms of superconductivity, let us note another research possibility in this direction. Doped strained STO is a good candidate for a polar metal and indeed is currently a topic of active study in multi-component metallic/dielectric heterostructures where STO is known to host a finite polarization [179]. Though such polar metals were predicted theoretically some time ago [180], recently there has been a resurgence of interest in such materials in part due to their anisotropic thermal and magnetoelectric properties [39, 181, 182]. At low temperatures such polar metals will surely become polar superconductors; such non-centrosymmetric superconductors are expected to have mixed-parity pairing mechanisms with topological aspects to their superconducting states [183].

These are just some of the many research questions that emerge from looking at paraelectrics and ferroelectrics at low temperatures; proximity to quantum phase transitions can be tuned by either pressure, stress, chemical or isotope substitution and perhaps even more. This is a rich area with plenty to explore, and we look forward to 
progress in these and many related topics.

\section{Acknowledgements}

We have benefitted from discussions with many colleagues including M. Alexe, K. Behnia, T. Birol, P. Coleman, M. Continentino, D. Khmelnitskii, P.B. Littlewood, L. Palova, K.M. Rabe, S.S. Saxena, Q. Si and C. Yu as this article has evolved. PC acknowledges Trinity College, Cambridge and the Cavendish Laboratory where this project was initiated. This work was supported by National Science Foundation grant NSF-DMR-1334428 (PC). GGL acknowledges support from grant no. EP/K012894/1 of the EPSRC and the CNPq/Science without Borders Program, and SER acknowledges support from a CONFAP Newton grant. PC and GGL also thank the Aspen Center for Physics and the National Science Foundation Grant No. PHYS-1066293 for hospitality where this work was further developed and discussed. 


\section{References}

[1] P. Hofman. Solid State Physics: An Introduction. Wiley-VCH, 2015.

[2] T.C. MacLeod, "Results from On-Orbit Testing of the FRAM Memory Test Experiment on the FASTSAT Microsatellite",. https://ntrs.nasa.gov/archiv/nasa/casi.ntrs.nasa.gov/20110015720.pdf. 2011.

[3] T.C. MacLeod, W.H. Sims, K.A. Varnavas, and F.D. Ho. Results from On-Orbit Testing of the FRAM Memory Test Experiment on the Fastsat Microsatellite. Integrated Ferroelectrics, 132:88-98, 2012.

[4] NPS' Newest Satellite Prepared for Launch. http://www.nps.edu/About/News/NPS-NewestSatellite-Prepared-for-Launch.html. 2016.

[5] Falcon Heavy. http://www.spacex.com/falcon-heavy. 2017.

[6] A.K. Larkin and D.E. Khmelnitskii. Phase Transitions in Uniaxial Ferroelectrics. Sov. Phys. JETP, 29:11231128, 1969.

[7] B.K. Chakrabati, A. Dutta, and P. Sen. Quantum Ising Phases and Transitions in Transverse Ising Models. Spring-Verlag, Berlin, 1996.

[8] G. Samara. Ferroelectrics Revisited - Advances in Materials and Physics. Solid State Physics, 56:239-381, 2001.

[9] O.E. Kvyatkovskii. Quantum Effects in Incipient and Low Temperature Ferroelectrics. Phys. Solid State, 43:1401, 2001.

[10] N.A. Spaldin, S.-W. Cheong, and R. Ramesh. Multiferroics: Past, Present and Future. Physics Today, 63:38-43, 2010.

[11] E.K.H. Salje. Ferroelastic materials. Ann. Rev. of Mater. Res., 42:265-283, 2012.

[12] S. Sachdev. Quantum Phase Transitions. Cambridge University Press, Cambridge, 1999.

[13] M. A. Continentino. Quantum Scaling in Many-Body Systems. World Scientific, Singapore, 2001.

[14] P. Gegenwart nad Q. Si and F. Steglich. Quantum criticality in heavy fermion metals. Nature Physics, 4:186-197, 2008.

[15] S. Sachdev. Quantum Magnetism and Criticality. Nature Physics, 4:173-185, 2008.

[16] A.B. Rechester. Contribution to the Theory of Second-Order Phase Tranistions at Low Temperatures. Sov. Phys. JETP, 33:423-430, 1971.

[17] D.E. Khmelnitskii and V.L. Shneerson. Phase Transition of the Displacement Type in Crystals at Very Low Temperature. Sov. Phys. JETP, 37:164-170, 1973.

[18] R. Roussev and A.J. Millis. Theory of the Quantum Paraelectric-Ferroelectric Transition. Physical Review B, 67:014105, 2003.

[19] P. Chandra L. Palova and P. Coleman. Quantum Critical Paraelectrics and the Casimir Effect in Time. Physical Review B, 79:075101, 2009.

[20] N. Das and S. Mishra. Fluctuations and Criticality in Quantum Paraelectrics. J. Phys. Cond. Mat., 21:095901, 2009.

[21] P. S. Riseborough. Quantum Fluctuations in Insulating Ferroelectrics. Chemical Physics, 375:184 $-186,2010$.

[22] S.E. Rowley, L.J. Spalek, R.P. Smith, M.P.M. Dean, M. Itoh, J.F. Scott, G.G. Lonzarich, and S.S. Saxena. Ferroelectric Quantum Criticality. Nature Physics, 10:367-372, 2014.

[23] T. Vojta. Quantum Phase Transitions. In Hoffman K.H. and M. Screiber, editors, Computational Statistical Physics. Springer-Verlag, Berlin, 2002.

[24] M. Vojta. Quantum phase transitions. Reports on Progress in Physics, 66:2069-2110, 2003.

[25] P. Coleman and A Schofield. Quantum criticality. Nature, 433:226-269, 2005.

[26] L.D. Landau and E.M. Lifshitz. Statistical Physics. Pergamon Press, Oxford, 1980, 1980.

[27] P. Coleman. Introduction to Many-Body Physics. Cambridge University Press, Cambridge, 2015.

[28] M.E. Lines and A.M. Glass. Principles and Applications of Ferroelectrics and Related Materials. Clarendon Press, Oxford, 1977. 
[29] P. Chandra and P.B. Littlewood. A Landau Primer for Ferroelectrics. In K.M. Rabe, C.H. Ahn, and J.M. Triscone, editors, Physics of Ferrolectrics: A Modern Perspective, pages 69-115. Springer-Verlag, Cambridge, 2007.

[30] C. Morice, P. Chandra, S. E. Rowley, G.G. Lonzarich, and S.S. Saxena. Hidden Fluctuations close to a Quantum Bicritical Point. arXiv:1611.0462, 2016.

[31] R.P. Feynman and A.R. Hibbs. Quantum Mechanics and Path Integrals. McGraw-Hill, 1965.

[32] E. Fatuzzo and W.J. Merz. Ferroelectricity. North-Holland, Amsterdam, 1967.

[33] F. Jona and G. Shirane. Ferroelectric Crystals. Dover Publications, New York, 1993.

[34] C. Kittel. Introduction to Solid State Physics. J. Wiley and Sons, Hoboken, 1996.

[35] B.A. Strukov and A.P. Levanyuk. Ferroelectric Phenomena in Crystals: Physical Foundations. Springer-Verlag, Berlin, 1998.

[36] J.F. Scott. Ferroelectric Memories. Springer-Verlag, Berlin, 2000.

[37] K. M. Rabe, M. Dawber, C. Lichtensteiger, C. H. Ahn, and J. M. Triscone. Modern Physics of Ferroelectrics: Essential Background. In K.M. Rabe, C.H. Ahn, and J.M. Triscone, editors, Physics of Ferrolectrics: A Modern Perspective, pages 1-29. Springer-Verlag, Cambridge, 2007.

[38] S. Tomic and M. Dressel. Ferroelectricity in Molecular Solids: A Review of Electrodynamic Properties. Reports of Progress in Physics, 78:096501, 2015.

[39] N. Benedek and T. Birol. 'Ferroelectric' Metals Reexamined: Fundamental Mechanisms and Design Considerations for New Materials. Journal of Materials Chemistry C, 4:4000-4015, 2016.

[40] Kensuke Kobayashi, Sachio Horiuchi, Reiji Kumai, Fumitaka Kagawa, Youichi Murakami, and Yoshinori Tokura. Electronic ferroelectricity in a molecular crystal with large polarization directing antiparallel to ionic displacement. Phys. Rev. Lett., 108:237601, Jun 2012.

[41] P.W. Anderson. On Ferroelectric Phase Transitions. In G. Skanavi, editor, Conf. Proc. Lebedev Physics Inst. USSR Acad. of Sci. Fizika Dielektrikov. Nova Science Publishers, 1960.

[42] W. Cochran. Crystal Stability and the Theory of Ferroelectricity. Advances in Physics, 9:387423, 1960.

[43] J.F. Scott. Soft-mode Spectroscopy: Experimental Studies of Structural Phase transitions. Rev. Modern Physics, 46:83-128, 1974.

[44] N.W. Ashcroft and N. David Mermin. Solid State Physics. Thomsen Learning, Toronto, 1976.

[45] K.M. Rabe and P. Ghosez. First-Principles Studies of Ferroelectrics Oxides. In K.M. Rabe, C.H. Ahn, and J.M. Triscone, editors, Physics of Ferrolectrics: A Modern Perspective, pages 117-172. Springer-Verlag, Cambridge, 2007.

[46] E. Cockayne and B.P. Burton. Phonons and Static Dielectric Constant in $\mathrm{CaTiO}_{3}$ from First Principles. Physical Review B, 62:3735-3743, 2000.

[47] E. Cockayne. First-principles Calculations of the Dielectric Properties of Perovskite-Type Materials. J. Eur. Ceramic. Soc., 23:2375-2379, 2003.

[48] C. H. Ahn, K. M. Rabe, and J.-M. Triscone. Ferroelectricity at the Nanoscale: Local Polarization in Oxide Thin Films and Heterostructures. Science, 303(5657):488-491, 2004.

[49] M. Born and K. Huang. Dynamical Theory of Crystal Lattices. Clarendon Press, Oxford, 1954.

[50] M.E. Fisher and A. Aharony. Dipolar Interactions at Ferromagnetic Critical Points. Phys. Rev. Lett, 30:559-562, 1973.

[51] A. Aharony. Dependence of Universal Critical Behavior on Symmetry and Range of interaction. In C. Domb and M.S. Green, editors, Phase Transitions and Critical Phenomena, vol. 6, pages 357-427. Academic Press, New York, 1976.

[52] G. Ahlers, A. Kornbilt, and H.J. Guggenheim. Logarithmic Corrections to the Landau Specific Heat near the Curie Temperature of the EDipolar Ising ferromagnet $L_{i T b F_{4}}$. Phys. Rev. Lett., 34:1227, 1975.

[53] E. Sandvold and E. Courtens. Logarithmic Correction of the Electric Susceptibility in Paraelectric Tris-Sarconsine Calcium Chloride. Phys. Rev. B, 27:5660, 1983.

[54] C. Domb. The Critical Point: A Historical Introduction to the Modern Theory of Critical 
Phenomena. Taylor and Francis, London, 1996.

[55] M.E. Fisher. Renormalization Group Theory: Its Basis and Formulation in Statistical Physics. Rev. Mod. Phys., 70:653-681, 1998.

[56] Shirane G., R. Nathans, and V.J. Mickiewicz. Temperature-Dependence of the Soft Ferroelectric Mode in $\mathrm{KTaO}_{3}$. Phys. Rev., 157:396-399, 1967.

[57] Yamada Y. and Shirane G. Neutron Scattering and Nature of the Soft Optical Phonon in $\mathrm{SrTiO}_{3}$. Phys. Soc. Jpn., 26:396-403, 1969.

[58] G. Shirane. Neutron Scattering Studies of Structural Phase Transitions at Brookhaven. Rev. Mod. Phys., 46:437-449, Jul 1974.

[59] D.E. Khmelnitskii and V.L. Shneerson. Low-temperature Displacement-Type Phase Transition in Crystals. Sov. Phys.-Solid State, 13:687, 1971.

[60] T. Schneider, H. Beck, and E. Stoll. Quantum effects in an $n$-component Vector model for Structural Phase Transitions. Phys. Rev. B, 13:1123-1130, Feb 1976.

[61] R. T. Brierley and P. B. Littlewood. Domain wall fluctuations in ferroelectrics coupled to strain. Phys. Rev. B, 89:184104, May 2014.

[62] J.H. Barrett. Dielectric Constant in Perovskite-Type Crystals. Physical Review B, 86:118-120, 1952.

[63] B. Wruck E.K.H. Salje and H. Thomas. Order-Parameter Saturation and Low Temperature Extension of Landau Theory. Z. Phys.B - Condens. Matter., 82:399-404, 1991.

[64] D.E. Khmelnitskii and V.L. Shneerson. Thermal Expansion and P-T Phase Diagram for a Displacement-Type Low-Temperature Phase Transition. Sov. Phys.-Solid State, 13:2158, 1971.

[65] D.E. Khmelnitskii. Critical Phenomenon in Deformation Phase Transition. Sov. Phys.-Solid State, 16:2079, 1975.

[66] L. Zhu, M. Garst, A. Rosch, and Q. Si. Universal Diverging Gruneisen Parameter and the Magnetocaloric Effect Close to Quantum Critical Points. Physical Review Letters, 91:66404, 2003.

[67] S.E. Rowley, J.C. Lashley, G.G. Lonzarich, J.F. Scott, and P. Chandra. Divergence of the Gruneisen Ratio at a Displacive Ferroelectric Quantum Critical Point, (Work in Progress). 2017.

[68] C. D. Batista, J. Schmalian, N. Kawashima, P. Sengupta, S.E. Sebastian, N. Harrison, M. Jaime, and I.R. Fisher. Geometrical Frustration and Dimensional Reduction at a Quantum Critical Point . Physical Review Letters, 98:257201, 2007.

[69] M. Jaime, V.F. Correa, N. Harrison, C.D. Bastista, N. Kawashima, Y. Kazuma, G.A. Jorge, R. Stern, I. Heinmaa, S.A. Zvyagi, Y. Sasago, and K. Uchinokura. Magnetic-Field-Induced Condensation of Triplons in Han Purple Pigment $\mathrm{BaCuSi}_{2} \mathrm{O}_{6}$. Physical Review Letters, 93:087203, 2004.

[70] T. Giamarchi, C. Ruegg, and O. Tchernyshyov. Bose-Einstein Condensation in Magnetic Insulators. Nature Physics, 4:198-204, 2008.

[71] C. Kraemer, N. Nikseresht, J.O. Piatek, N. Tsyrulin, B.D. Piazza, K. Kiefer, B. Klemke, T.F. Rosenbaum, G. Aeppli, C. Gannarelli, K. Prokes, A. Podlesnyak, T. Strassle, L. Keller, O. Zaharko, K.W. Kramer, and H.M. Rorrow. Dipolar Antiferromagnetism and Quantum Criticality in $\mathrm{LiErF}_{4}$. Science, 336:1416-1419, 2012.

[72] P. Canfield. Fishing the Fermi Sea. Nature Physics, 4:167-169, 2008.

[73] V.V. Lemanov. Improper Ferroelastic $\mathrm{SrTiO}_{3}$ and What We Know Today about Its Properties. Ferroelectrics, 265:1-21, 2002.

[74] J. Dec, W. Kleeman, K. Boldyreva, and M. Itoh. Unique Features of Strontium Titanate. Ferroelectrics, 314:7-18, 2005.

[75] E.K.H. Salje, O. Aktas, M.A. Carpenter, and J.F. Scott. Domains within Domains and Walls within Walls: Evidence for Polar Domains in Cryongenic $\mathrm{SrTiO}_{3}$. Physical Review Letters, 111:247603, 2013.

[76] H. J. Harsan Ma, S. Scharinger, S. W. Zeng, D. Kohlberger, M. Lange, A. Stöhr, X. Renshaw 
Wang, T. Venkatesan, R. Kleiner, J. F. Scott, J. M. D. Coey, D. Koelle, and Ariando. Local Electrical Imaging of Tetragonal Domains and Field-Induced Ferroelectric Twin Walls in Conducting $\mathrm{SrTiO}_{3}$. Phys. Rev. Lett., 116:257601, Jun 2016.

[77] S. E. Rowley and G. G. Lonzarich. Vortex Physics: Ferroelectrics in a Twist. Nature Physics, 10:907-908, 2014.

[78] S.A. Hayward, F.D. Morrison, and J.F. Scott. Interaction between quantum paraelectricity and ferroelasticity in $\mathrm{SrTiO}_{3}$. Journal of Physics: Condensed Matter, 17(43):7009, 2005.

[79] K. A. Müller and H. Burkard. $\mathrm{SrTiO}_{3}$ : An Intrinsic Quantum Paraelectric Below 4 K. Phys. Rev. B, 19:3593-3602, Apr 1979.

[80] R. Martoňák and E. Tosatti. Path-integral Monte Carlo study of a Model Two-Dimensional Quantum Paraelectric. Phys. Rev. B, 49:12596-12613, May 1994.

[81] W. Zhong and David Vanderbilt. Effect of quantum fluctuations on structural phase transitions in $\mathrm{SrTiO}_{3}$ and $\mathrm{BaTiO}_{3}$. Phys. Rev. B, 53:5047-5050, Mar 1996.

[82] G. Conduit and B. D. Simons. Theory of Quantum Paraelectrics and the Metaelectric Transition. Physical Review B, 81:024102, 2010.

[83] Mitsuru Itoh and Ruiping Wang. Quantum ferroelectricity in srtio $o_{3}$ induced by oxygen isotope exchange. Applied Physics Letters, 76(2):221-223, 2000.

[84] Ruiping Wang and Mitsuru Itoh. Suppression of the quantum fluctuation in ${ }^{18}$ O-enriched strontium titanate. Phys. Rev. B, 64:174104, Oct 2001.

[85] J. F. Scott, J. Bryson, M. A. Carpenter, J. Herrero-Albillos, and M. Itoh. Elastic and Anelastic Properties of Ferroelectric $\mathrm{SrTi}^{18} \mathrm{O}_{3}$ in the kHz-MHz Regime. Phys. Rev. Lett., 106:105502, Mar 2011.

[86] A Bussmann-Holder, H Büttner, and A R Bishop. Stabilization of ferroelectricity in quantum paraelectrics by isotopic substitution. Journal of Physics: Condensed Matter, 12(6):L115, 2000.

[87] M. Itoh, T. Yagi, Y. Uesu, W. Kleemann, and R. Blinc. Phase transition and random-field induced domain wall response in quantum ferroelectrics $\mathrm{SrTi}^{18} \mathrm{O}_{3}$ : review and perspective. Science and Technology of Advanced Materials, 5(4):417, 2004.

[88] A. Bussmann-Holder and A. R. Bishop. Dimensional crossover and absence of quantum criticality in $\operatorname{SrTi}^{16} \mathrm{O}_{1-x}^{18} O_{x}$. Phys. Rev. B, 78:104117, Sep 2008.

[89] E.I. Venturini, G.A. Samara, M. Itoh, and R. Wang. Pressure as a Probe of the Physics of ${ }^{18} \mathrm{O}$-subsituted $\mathrm{SrTiO}_{3}$. Physical Review B, 69:184105, 2004.

[90] J. G. Bednorz and K. A. Müller. $\mathrm{Sr}_{1-x} \mathrm{Ca}_{x} \mathrm{TiO} \mathrm{O}_{3}$ : An XY Quantum Ferroelectric with Transition to Randomness. Phys. Rev. Lett., 52:2289-2292, Jun 1984.

[91] C.W. Rischau, X. Lin, C.P. Grams, D. Finck, S. Harms, J. Engelmayer, T. Lorenz, Y. Gallais, B. Fauque, J. Hemberger, and K. Behnia. A Ferroelectric Quantum Phase Transition inside the Superconducting dome of $\mathrm{Sr}_{1-x} \mathrm{Ca}_{x} \mathrm{TiO}_{3-\delta}$. Nature Physics, 2017.

[92] Daniel E. Grupp and Allen M. Goldman. Giant Piezoelectric Effect in Strontium Titanate at Cryogenic Temperatures. Science, 276(5311):392-394, 1997.

[93] M. Takesada, T. Yagi, M. Itoh, and S.Y. Koshihara. A Giant Photoinduced Dielectric Constant of Quantum Perovskite Oxides Observed under a weak DC Electric Field. J. Phys. Soc. Japan, 72:37-40, 2003.

[94] T. Hasegawa, S.I. Mouri, Y. Yamada, and K. Tanaka. Giant Photoinduced Dielecticity in $\mathrm{SrTiO}_{3}$ . J. Phys. Soc. Japan, 72:41-44, 2003.

[95] Keiichiro Nasu. Photogeneration of superparaelectric large polarons in dielectrics with soft anharmonic $T_{1 u}$ phonons. Phys. Rev. B, 67:174111, May 2003.

[96] W. Kleeman, J. Dec, Y.G. Wang, P. Lehnen, and S.A. Prosandeev. Phase Transitions and Relaxor Properties of Doped Quantum Paraelectrics. Journal of Physics and Chemistry of Solids, 61:167-176, 2000.

[97] Chen Ang, Zhi Yu, P. M. Vilarinho, and J. L. Baptista. Bi: $\mathrm{SrTiO}_{3}$ : A quantum ferroelectric and a relaxor. Phys. Rev. B, 57:7403-7406, Apr 1998.

[98] A. Spinelli, M. A. Torija, C. Liu, C. Jan, and C. Leighton. Electronic transport in doped $\mathrm{SrTiO}_{3}$ : 
Conduction mechanisms and potential applications. Phys. Rev. B, 81:155110, Apr 2010.

[99] J. Son, P. Moetakef, B. Jalan, O. Bierwagen, N.J. Wright, R. Engel-Herbert, and S. Stemmer. Epitaxial $\mathrm{SrTiO}_{3}$ Films with Electron Mobilities Exceeding $30,000 \mathrm{~cm}^{2} \mathrm{~V}^{-1} \mathrm{~s}^{-1}$. Nature Materials, 9:482, 2010.

[100] K. Behnia. On Mobility of Elecrons in a Shallow Fermi Sea over a Rough SeaFloor. J. Phys..: Cond. Mat., 27:375501, 2015.

[101] X. Lin, B. Fauque, and K. Behnia. Scalable $T^{2}$ Resistivity in a Small Single-Component Fermi Surface. Science, 349:945, 2015.

[102] Y. Kozuka, T. Susaki, and H. Y. Hwang. Vanishing Hall Coefficient in the Extreme Quantum Limit in Photocarrier-Doped $\mathrm{SrTiO}_{3}$. Phys. Rev. Lett., 101:096601, Aug 2008.

[103] Xiao Lin, Zengwei Zhu, Benoît Fauqué, and Kamran Behnia. Fermi Surface of the Most Dilute Superconductor. Phys. Rev. X, 3:021002, Apr 2013.

[104] Om Prakash, Anil Kumar, A. Thamizhavel, and S. Ramakrishnan. Evidence for bulk superconductivity in pure bismuth single crystals at ambient pressure. Science, 2016.

[105] P. Gallagher, M. Lee, J.R. Williams, and D. Goldhaber-Gordon. Gate-tunable Superconducting Weak Link and Quantum Point Contact Spectroscopy on a Strontium Titanate Surface. Nature Physics, 10:748, 2014.

[106] Jonathan M. Edge, Yaron Kedem, Ulrich Aschauer, Nicola A. Spaldin, and Alexander V. Balatsky. Quantum critical origin of the superconducting dome in srtio $_{3}$. Phys. Rev. Lett., 115:247002, Dec 2015.

[107] Yaron Kedem, Jian-Xin Zhu, and Alexander V. Balatsky. Unusual superconducting isotope effect in the presence of a quantum criticality. Phys. Rev. B, 93:184507, May 2016.

[108] A. Stucky, G.W. Scheerer, Z. Ren, D. Jaccard, J.-M. Poumirol, C. Barretaeau, E. Giannini, and D. van der Marel. Isotope effect in superconducting n-doped srtio 3 . Sci. Reports, 6:37582, 2016.

[109] A. Ohtomo and H. Y. Hwang. A High-Mobility Electron Gas at the $\mathrm{LaAlO}_{3} / \mathrm{SrTiO}_{3}$ Heterointerface. Nature, 427:423, 2004.

[110] S. Lerer, M. Ben Shalom, G. Deutscher, and Y. Dagan. Low-temperature dependence of the thermomagnetic transport properties of the $\mathrm{SrTiO}_{3} / \mathrm{LaAlO}_{3}$ interface. Phys. Rev. B, 84:075423, Aug 2011.

[111] J.A. Sulpizio, S. Ilani, P. Irvn, and J. Levy. Nanoscale Phenomena in Oxide Heterostructures. Annual Review of Materials Research, 44:117-149, 2014.

[112] J.-F. Ge, Z.-L. Liu, C. Liu, C.-L. Gao, D. Qian, Q.-K. Xue, Y. Liu, and J.-F. Jia. Superconductivity above $100 \mathrm{~K}$ in single-layer $\mathrm{FeSe}$ films on doped $\mathrm{SrTiO}_{3}$. Nature Mater., 14:285, 2015.

[113] T. Ishidate, S. Abe, H. Takahashi, and N. Môri. Phase Diagram of $\mathrm{BaTiO}_{3}$. Phys. Rev. Lett., 78:2397-2400, Mar 1997.

[114] S. Horiuchi, Y. Okimoto, R. Kumai, and Y. Tokura. Quantum Phase Transition in Organic Charge-Transer Complexes. Science, 299:229, 2003.

[115] S. Horiuchi, K. Kobayashi, R. Kumai, N. Minami, F. Kagawa, and Y. Tokura. Quantum Ferroelectricity in Charge-Transfer Complex Crystals. Nature Communications, 6:7469, 2015.

[116] S.E. Rowley, M. Hadjimichael, M.N. Ali, Y.C. Durmaz, J.C Lasley, R.J. Cava, and J.F. Scott. Quantum Criticality in a Uniaxial Organic Ferroelectric. J. Phys.: Condens. Matt., 27:395901, 2015.

[117] T. Suski, S. Takaoka, K. Murase, and S. Porowski. New Phenomena of Low Temperature Resistivity Enhancement in Quantum Ferroelectric Semiconductors. Solid State Communications, 45:259-262, 1983.

[118] J.F. Scott. Applications of Modern Ferroelectrics. Science, 315:954-959, 2007.

[119] Su-Ting Han, Ye Zhou, and V. A. L. Roy. Towards the Development of Flexible Non-Volatile Memories. Advanced Materials, 25(38):5425-5449, 2013.

[120] J.F. Scott. Future Issues in Ferroelectric Miniaturization. Ferroelectrics, 206-07:365-379, 1998. 
[121] M. Alexe, "Nanoscale Ferroelectrics". Invited presentation, 10th European Meeting on Ferroelectricity. 2003.

[122] S.-G. Lu and Q. Zhang. Electrocaloric Materials for Solid-State Refrigeration. Advanced Materials, 21:1983-87, 2009.

[123] J.F. Scott. Electrocaloric Materials. Annu. Rev. Mater. Res., 41:229-240, 2011.

[124] I. Takeuchi and K. Sandeman. Solid State Cooling wtih Caloric Materials. Physics Today, 68:48-54, 2015.

[125] A.S. Mischenko, W. Zhang, J.F. Scott, R.W. Whatmore, and N.D. Mathur. Giant Electrocaloric Effect in Thin-Film $\mathrm{PbZr}_{0.95} \mathrm{Ti}_{0.05} \mathrm{O}_{3}$. Science, 311:1270-1, 2006.

[126] W. N. Lawless. Specific heat and electrocaloric properties of $\mathrm{KTaO}_{3}$ at low temperatures. Phys. Rev. B, 16:433-439, Jul 1977.

[127] W. N. Lawless and A.J. Morrow. Specific heat and electrocaloric properties of a $\mathrm{SrTiO}_{3}$ ceramic at low temperatures. Ferroelectrics, 15:159-65, 1977.

[128] R. Radebaugh, W.N. Lawless, J.D. Siegwarth, and A.J. Morrow. Feasibility of Electrocaloric Refrigeration for the 4-15 K Temperature Range. Cryogenics, 19:187-208, 1979.

[129] R. Radebaugh, W.N. Lawless, J.D. Siegwarth, and A.J. Morrow. Electrocaloric refrigeration at cryogenic temperatures. Ferroelectrics, 27:205-11, 1980.

[130] M. E. Zhitomirsky. Enhanced magnetocaloric effect in frustrated magnets. Phys. Rev. B, 67:104421, Mar 2003.

[131] B Wolf, A. Honecker, W. Hofstetter, U. Tutsch, and M. Lang. Cooling Through Quantum Criticality and Many-Body Effects in Condensed Matter and Cold Gases. International Journal of Physics, 28:1430017, 2014.

[132] P. Chandra, G. Lonzarich, Rowley S., and J.F. Scott. Electrocaloric Cooling at a Quantum Tricritical Lifshitz Point (Work in Progress). 2017.

[133] I. Shepherd and G. Feher. Cooling by Adiabatic Depolarization of $\mathrm{OH}^{-}$Molecules in KCl. Phys. Rev. Lett., 15:194-198, Aug 1965.

[134] H. B. Shore. Model Hamiltonian for Paraelectric Impurities in Alkali Halides. Phys. Rev., 151:570-577, Nov 1966.

[135] W.N. Lawless. Thermodynamics of Electrocaloric Phenomena in KCl:OH. Paraelectric Cooling. J. Phys. Chem. Solids, 30:1161-1172, 1969.

[136] R. O. Pohl, V. L. Taylor, and W. M. Goubau. Electrocaloric Effect in Doped Alkali Halides. Phys. Rev., 178:1431-1436, Feb 1969.

[137] P.J. Shirron. Cooling Capabilities of Adiabatic Demagnetization Refrigerators. J.Low Temp. Phys., 148:915-920, 2007.

[138] R. Sayyah, T.C. Macleod, and F.D. Ho. Radiation-Hardened Electronics and Ferroelectric Memory for Space Flight Systems. Ferroelectrics, 413(1):170-175, 2011.

[139] M. Hoffmann, U. Schroder, C. Kunneth, Kersch A., S. Starschich, U. Bottger, and T. Mikolajick. Ferroelectric Phase Transitions in Nanoscale $\mathrm{HfO}_{2}$ films enable giant pyroelectric energy conversions and highly efficient supercapacitors. NanoEnergy, 18:154-164, 2015.

[140] S. K. Mishra, N. Choudhury, S. L. Chaplot, P. S. R. Krishna, and R. Mittal. Competing antiferroelectric and ferroelectric interactions in $\mathrm{NaNbO}_{3}$ : Neutron diffraction and theoretical studies. Phys. Rev. B, 76:024110, Jul 2007.

[141] S.I. Raevskaya, I.P. Raevski, S.P. Kubrin, M.S. Panchelyuga, V.G. Smotrakov, V.V. Eremkin, and S.A. Prosandeev. Quantum Paraelectricity Coexisting with a Ferroelectric Metastable State in Single Crystals of $\mathrm{NaNbO}_{3}$ : A New Quantum Effect. J. Phys.: Condens. Matter, 20:232202, 2008.

[142] S.-P. Shen, J.-C. Wu, J.-D. Song, X.-F. Sun, Y.-F. Yang, Y.-S. Chai, D.-S. Shang, S.-G. Wnag, J.F. Scott, and Young Sun. Quantum Electric-Dipole Liquid on a Triangular Lattice. Nature Comm., 7:10569, 2016.

[143] J.F. Scott. Ferroelectrics: Novel Geometric Ordering of Ferroelectricity. Nature Materials, 4:1314,2005 . 
[144] I.I. Naumov, L. Beallaiche, and H. Fu. Unusual Phase Transitions in Ferroelectric Nanodisks and Nanorods. Nature, 432(5):737-740, 2004.

[145] M Dawber, A Gruverman, and J F Scott. Skyrmion model of nano-domain nucleation in ferroelectrics and ferromagnets. Journal of Physics: Condensed Matter, 18(5):L71, 2006.

[146] Liliana Goncalves-Ferreira, Simon A. T. Redfern, Emilio Artacho, and Ekhard K. H. Salje. Ferrielectric Twin Walls in $\mathrm{CaTiO}_{3}$. Phys. Rev. Lett., 101:097602, Aug 2008.

[147] Shi-Zeng Lin, Xueyun Wang, Yoshitomo Kamiya, Gia-Wei Chern, Fei Fan, David Fan, Brian Casas, Yue Liu, Valery Kiryukhin, Wojciech H. Zurek, Cristian D. Batista, and SangWook Cheong. Topological Defects as Relics of Emergent Continuous Symmetry and Higgs Condensation of Disorder in Ferroelectrics. Nature Physics, 10:970-977, 2014.

[148] J.F. Scott. Searching for new Ferroelectrics and Multiferroics: A User's Point of View. Computational Materials, 1:15006, 2015.

[149] Shuai Dong, Jun-Ming Liu, Sang-Wook Cheong, and Zhifeng Ren. Multiferroic materials and magnetoelectric physics: symmetry, entanglement, excitation, and topology. Advances in Physics, 64(5-6):519-626, 2015.

[150] T. Katsufuji and H. Takagi. Coupling between magnetism and dielectric properties in quantum paraelectric EuTiO 3 . Phys. Rev. B, 64:054415, Jul 2001.

[151] Jae Wook Kim, S. Y. Haam, Y. S. Oh, S. Park, S.-W. Cheong, P. A. Sharma, M. Jaime, N. Harrisonc, Jung Hoon Han, Gun-Sang Jeon, P. Coleman, and Kee Hoon Kim. Observation of a Multiferroic Critical Ed Point. Proc. Nat. Acad., 106:15574-76, 2016.

[152] N. Das. Quantum Critical Behavior of a Magnetic Quantum Paraelectric. Physics Letters A, 376:2683-2687, 2012.

[153] Jason Schiemer, Leszek J. Spalek, Siddharth S. Saxena, Christos Panagopoulos, Takuro Katsufuji, Annette Bussmann-Holder, Jürgen Köhler, and Michael A. Carpenter. Magnetic field and in situ stress dependence of elastic behavior in $\mathrm{EuTiO}_{3}$ from resonant ultrasound spectroscopy. Phys. Rev. B, 93:054108, Feb 2016.

[154] S. E. Rowley, Y.-S. Chai, S.-P. Shen, Y.Sun, A.T. Jones, B.E. Watts, and J.F. Scott. Uniaxial Ferroelectric Quantum Criticality in Multiferroic Hexaferrites $\mathrm{BaFe}_{12} \mathrm{O}_{19}$ and $\mathrm{SrFe}_{12} \mathrm{O}_{19}$. Scientific Reports, 6:1-5, 2016.

[155] Carlos A. F. Vaz, Jason Hoffman, Charles H. Ahn, and Ramamoorthy Ramesh. Magnetoelectric coupling effects in multiferroic complex oxide composite structures. Advanced Materials, 22(2627):2900-2918, 2010.

[156] C A F Vaz. Electric field control of magnetism in multiferroic heterostructures. Journal of Physics: Condensed Matter, 24(33):333201, 2012.

[157] A. Midya, P. Mandal, Km. Rubi, Ruofan Chen, Jiang-Sheng Wang, R. Mahendiran, G. Lorusso, and M. Evangelisti. Large adiabatic temperature and magnetic entropy changes in $\mathrm{EuTio}_{3}$. Phys. Rev. B, 93:094422, Mar 2016.

[158] N.F. Mott. Metal-Insulator Transitions (2nd Edition). Taylor and Francis, London, 1990.

[159] Peter Phillip Edwards and Michell J. Sienko. Universality aspects of the metal-nonmetal transition in condensed media. Phys. Rev. B, 17:2575-2581, Mar 1978.

[160] K. Behnia. Private Communication. 2014.

[161] T. Okuda, K. Nakanishi, S. Miyasaka, and Y. Tokura. Large thermoelectric response of metallic perovskites: $\mathrm{Sr}_{1-x} \mathrm{La}_{x} \mathrm{TiO}_{3}(0 \leq x \leq 0.1$. Phys. Rev. B, 63:113104, Mar 2001.

[162] J.R. Schrieffer. Theory of Superconductivity. Benjamin/Cummings, London, 1983.

[163] L.V. Gurevich, A.I. Larkin, and Y.A. Firsov. On the Possibility of Superconductivity in Sermiconductors. Sov. Phys. Sol. State, 4:131, 1962.

[164] Marvin L. Cohen. The Existence of a Superconducting State in Semiconductors. Rev. Mod. Phys., 36:240-243, Jan 1964.

[165] Marvin L. Cohen. Superconductivity in many-valley semiconductors and in semimetals. Phys. Rev., 134:A511-A521, Apr 1964.

[166] J. F. Schooley, W. R. Hosler, and Marvin L. Cohen. Superconductivity in Semiconducting 
$\mathrm{SrTiO}_{3}$. Phys. Rev. Lett., 12:474-475, Apr 1964.

[167] M.L. Cohen. Superconductivity in low-carrier density systems: Degenerate semiconductors. In R.D. Parks, editor, Superconductivity, pages 615-664. Marcel Dekker, New York, 1969.

[168] R. M. Fernandes, J. T. Haraldsen, P. Wölfle, and A. V. Balatsky. Two-band superconductivity in doped $\mathrm{SrTiO}_{3}$ films and interfaces. Phys. Rev. B, 87:014510, Jan 2013.

[169] Jonathan Ruhman and Patrick A. Lee. Superconductivity at very low density: The case of strontium titanate. Phys. Rev. B, 94:224515, Dec 2016.

[170] L.P. Gorkov. Phonon Mechanism in the most dilute superconductor n-type $\mathrm{SrTiO}_{3}$. Proc. Nat. Acad., 113:4646-4651, 2016.

[171] L.P. Gorkov. Back to the Mechanism of Superconductivity in Low-Doped $\mathrm{SrTiO}_{3}$. arXiv:1610.02062, 2016.

[172] J. F. Schooley, W. R. Hosler, E. Ambler, J. H. Becker, Marvin L. Cohen, and C. S. Koonce. Dependence of the Superconducting Transition Temperature on Carrier Concentration in Semiconducting $\mathrm{SrTiO}_{3}$. Phys. Rev. Lett., 14:305-307, Mar 1965.

[173] C. S. Koonce, Marvin L. Cohen, J. F. Schooley, W. R. Hosler, and E. R. Pfeiffer. Superconducting Transition Temperatures of Semiconducting $\mathrm{SrTiO}_{3}$. Phys. Rev., 163:380-390, Nov 1967.

[174] G. Binnig, A. Baratoff, H. E. Hoenig, and J. G. Bednorz. Two-Band Superconductivity in NbDoped $\mathrm{SrTiO}_{3}$. Phys. Rev. Lett., 45:1352-1355, Oct 1980.

[175] H. Suzuki, H. Bando, Y. Ootuka, I. H. Inoue, T. Yamamoto, K. Takahashi, and Y. Nishihara. Superconductivity in Single-Crystalline $\mathrm{Sr}_{1-x} \mathrm{La}_{x} \mathrm{TiO}_{3}$. Journal of the Physical Society of Japan, 65(6):1529-1532, 1996.

[176] J. Biscaras, N. Bergeal, A. Kushwaha, T. Wolf, A. Rastogi, R.C. Budhani, and J. Lesueur. Twodimensional superconductivity at a Mott insulator/band insulator interface $\mathrm{LaTiO}_{3} / \mathrm{SrTiO}_{3}$. Nature Comm., 1:89, 2010.

[177] Pouya Moetakef, James R. Williams, Daniel G. Ouellette, Adam P. Kajdos, David GoldhaberGordon, S. James Allen, and Susanne Stemmer. Carrier-Controlled Ferromagnetism in $\mathrm{SrTiO}_{3}$. Phys. Rev. X, 2:021014, Jun 2012.

[178] Yun-Yi Pai, Anthony Tylan-Tyler, Patrick Irvin, and Jeremy Levy. Physics of srtio -based $_{3}$ heterostructures and nanostructures: A reivew. arXiv:1706.07690, XX, 2017.

[179] S.Y. Park, Y. Cao, K Rabe, and J. Chakhalian. Polar Metallic Phase in $\mathrm{BaTiO}_{3} / \mathrm{SrTiO}_{3} / \mathrm{LaTiO}_{3}$, (Work in Progress). 2017.

[180] P. W. Anderson and E. I. Blount. Symmetry Considerations on Martensitic Transformations: "Ferroelectric" Metals? Phys. Rev. Lett., 14:217-219, Feb 1965.

[181] M. Lezaic. Clockwork at the Atomic Scale. Nature, 533:38, 2016.

[182] T.H. Kim, D. Puggioni, Y. Yuan, L. Xie, H. Zhou, N. Campbell, P.J. Ryan, Y. Choi, J.-W. Ki, J.R. Patzner, S. Ryu, J.P. Podkaminer, J. Irwin, Y. Ma, C.J. Fennie, M.S. Rzchowski, X.Q. Pan, V. Gopalan, J.M. Rondinelli, and C.B. Eom. Polar Metals by Geometric Design. Nature, 533:68-71, 2016.

[183] S. Yip. Noncentrosymmetric Superconductors. Ann. Rev. Cond. Matt. Phys., 5:15-33, 2014. 